\title{
Pacific
}

Journal of

Mathematics

\section{EIGENVARIETIES AND INVARIANT NORMS}

Claus M. Sorensen

Volume $275 \quad$ No. 1

May 2015 


\title{
EIGENVARIETIES AND INVARIANT NORMS
}

\author{
Claus M. Sorensen
}

We give a proof of the Breuil-Schneider conjecture in a large number of cases, which complement the indecomposable case, which we dealt with earlier. In this paper, we view the conjecture from a broader global perspective. If $U_{/ F}$ is any definite unitary group, which is an inner form of GL(n) over $\mathcal{K}$, we point out how the eigenvariety $\mathbb{X}\left(K^{p}\right)$ parametrizes a global $\boldsymbol{p}$-adic Langlands correspondence between certain $\boldsymbol{n}$-dimensional $\boldsymbol{p}$ adic semisimple representations $\rho$ of $\operatorname{Gal}(\overline{\mathbb{Q}} \mid \mathcal{K})$ (or what amounts to the same, pseudorepresentations) and certain Banach-Hecke modules $\mathcal{B}$ with an admissible unitary action of $U\left(F \otimes \mathbb{Q}_{p}\right)$, when $p$ splits. We express the locally regular-algebraic vectors of $\mathcal{B}$ in terms of the Breuil-Schneider representation of $\rho$. As an application, we give a weak form of local-global compatibility in the crystalline case, showing that the Banach space representations $\boldsymbol{B}_{\xi, \zeta}$ of Schneider and Teitelbaum fit the picture as predicted.

1. Introduction

191

2. Automorphic Galois representations 201

3. Completed cohomology 206

4. Eigenvarieties 210

5. Banach space representations 214

6. Compatibility with classical local Langlands 216

7. Zariski density of crystalline points 221

Acknowledgements 228

References $\quad 228$

\section{Introduction}

Let $\mathcal{K}$ be a number field. The Fontaine-Mazur conjecture [1995] predicts a characterization of all (irreducible) Galois representations $r: \Gamma_{\mathcal{K}}=\mathrm{Gal}(\overline{\mathbb{Q}} \mid \mathcal{K}) \rightarrow \mathrm{GL}_{n}\left(\mathbb{Q}_{p}\right)$ occurring naturally - by which we mean some Tate twist of $r$ is a subquotient of the étale cohomology $H^{\bullet}\left(X, \mathbb{Q}_{p}\right)$ of some smooth projective variety $X_{/ \mathcal{K}}$. It is a major result (due to Tsuji and others) that every such $r$ is geometric, which means it is unramified at all but finitely many places, and potentially semistable at all places

MSC2010: 11F33.

Keywords: Galois representations, p-adic Langlands, eigenvarieties, automorphic forms. 
above $p$. Fontaine and Mazur assert the converse: that every geometric $r$ occurs in cohomology (up to a Tate twist). The potentially semistable representations are now more or less completely understood, by work of Colmez and Fontaine [2000]. They are given by admissibly filtered $(\phi, N)$-modules (with Galois action), which are objects of a more concrete combinatorial nature. The $p$-adic Langlands program, still in its initial stages, attempts to link $p$-adic Hodge theory with nonarchimedean functional analysis. Locally, if $K$ is a fixed finite extension of $\mathbb{Q}_{p}$, and $L \mid \mathbb{Q}_{p}$ is another sufficiently large finite extension (the coefficient field), one hopes to pair certain Galois representations $r: \Gamma_{K} \rightarrow \mathrm{GL}_{n}(L)$ with certain Banach $L$-spaces with a unitary admissible $\mathrm{GL}_{n}(K)$-action. This is now well understood for $\mathrm{GL}_{2}\left(\mathbb{Q}_{p}\right)$ thanks to recent work of Berger, Breuil, Colmez, Paskunas, and others. See [Berger 2011] for a nice survey.

The goal of this paper is to shed some light on a global analogue, for any $n$, and any $\mathrm{CM}$ field $\mathcal{K}$. To give the flavor, if $\mathcal{K} \mid \mathbb{Q}$ is a quadratic imaginary field in which $p$ splits, we will set up a bijection between certain Galois representations $r: \Gamma_{\mathcal{K}} \rightarrow \mathrm{GL}_{n}(L)$ (actually, pseudorepresentations) and certain Banach-Hecke modules with a unitary admissible $\mathrm{GL}_{n}\left(\mathbb{Q}_{p}\right)$-action. This is most likely folklore. We emphasize that this bijection is based on matching Satake parameters and Frobenius eigenvalues at places away from $p$. More importantly, we relate the algebraic vectors to the $p$-adic Hodge theory on the Galois side. The word certain here has a precise meaning. It means those representations which come from an eigenvariety, of some fixed tame level $K^{p}$. We will be precise below.

We model the discussion on the $\mathrm{GL}_{2}\left(\mathbb{Q}_{p}\right)$-case: To any continuous Galois ${ }^{1}$ representation $\rho: \Gamma_{\mathbb{Q}_{p}} \rightarrow \mathrm{GL}_{2}(L)$, the $p$-adic Langlands correspondence associates a unitary Banach $L$-space representation $B(\rho)$ of $\mathrm{GL}_{2}\left(\mathbb{Q}_{p}\right)$. Moreover, $\rho$ is de Rham with distinct Hodge-Tate weights precisely when there are nonzero locally algebraic vectors: $B(\rho)^{\text {alg }} \neq 0$. Here we use the notation from [Breuil 2010, p. 7]. Some authors prefer to write $B(\rho)^{1-a l g}$. (Recall that a vector is locally algebraic if some open subgroup acts polynomially. They were studied in detail in [Schneider and Teitelbaum 2001]. See Section 3.2 below for more details.) Conversely, if $\rho$ is de Rham with (distinct) Hodge-Tate weights, $\{0,1-k\}$ (with the convention that the cyclotomic character has weight -1 ), then the locally algebraic vectors are given by

$$
B(\rho)^{\text {alg }}=\operatorname{Sym}^{k-2}\left(L^{2}\right) \otimes \pi(\rho)
$$

for a smooth generic representation $\pi(\rho)$, possibly reducible, obtained by a slight modification of the classical local Langlands correspondence.

The Breuil-Schneider conjecture. The local $p$-adic Langlands program is somewhat vague, and a precise conjectural framework is still developing beyond the case

\footnotetext{
${ }^{1}$ In the introduction, we will denote global Galois representations by $r$, local ones by $\rho$.
} 
of $\mathrm{GL}_{2}\left(\mathbb{Q}_{p}\right)$, where pretty much everything is known. However, there is a weak (but precise) version formulated in [Breuil and Schneider 2007], which we now recall. We keep our finite extension $K \mid \mathbb{Q}_{p}$, and a finite Galois extension thereof, $K^{\prime} \mid K$. Pick a third field of coefficients $L \subset \overline{\mathbb{Q}}_{p}$, finite over $\mathbb{Q}_{p}$, but large enough so that it contains the Galois closures of $K$ and $K_{0}^{\prime}$ (the maximal unramified subfield of $K^{\prime}$ ). The roles of these fields are the following. We consider potentially semistable representations $\rho: \Gamma_{K} \rightarrow \mathrm{GL}_{n}(L)$, which become semistable when restricted to $\Gamma_{K^{\prime}}$. As mentioned above, such $\rho$ correspond to $(\phi, N) \times \mathrm{Gal}\left(K^{\prime} \mid K\right)$-modules $D$ with an admissible filtration. This makes use of Fontaine's period ring $B_{\text {st }}$,

$$
D=\left(B_{\mathrm{st}} \otimes_{\mathbb{Q}_{p}} \rho\right)^{\Gamma_{K^{\prime}}} .
$$

This is a finite, free $K_{0}^{\prime} \otimes_{\mathbb{Q}_{p}} L$-module of rank $n$ with a semilinear Frobenius $\phi$, a (nilpotent) monodromy operator $N$ such that $N \phi=p \phi N$, a commuting action of $\operatorname{Gal}\left(K^{\prime} \mid K\right)$, and an admissible Galois-stable filtration on $D_{K^{\prime}}$. Note

$$
K^{\prime} \otimes_{\mathbb{Q}_{p}} L \simeq \prod_{\tau \in \operatorname{Hom}(K, L)} K^{\prime} \otimes_{K, \tau} L .
$$

Accordingly, $D_{K^{\prime}} \simeq \prod_{\tau} D_{K^{\prime}, \tau}$, and each $K^{\prime} \otimes_{K, \tau} L$-module $D_{K^{\prime}, \tau}$ is filtered.

- Hodge-Tate numbers. For each $\tau: K \hookrightarrow L$, we let $i_{1, \tau} \leq \cdots \leq i_{n, \tau}$ denote the jumps in the Hodge filtration (listed with multiplicity). That is,

$$
\operatorname{gr}^{i}\left(D_{K^{\prime}, \tau}\right) \neq 0 \Longleftrightarrow i \in\left\{i_{1, \tau}, \ldots, i_{n, \tau}\right\} .
$$

We will denote this multiset of integers by $H T_{\tau}(\rho)=\left\{i_{j, \tau}: j=1, \ldots, n\right\}$.

- Weil-Deligne representation. If we forget about the filtration, the resulting $(\phi, N) \times \operatorname{Gal}\left(K^{\prime} \mid K\right)$-module corresponds to a Weil-Deligne representation, once we fix an embedding $K_{0}^{\prime} \hookrightarrow L$; see Proposition 4.1 in [Breuil and Schneider 2007] for details on this correspondence. Basically, one looks at the $n$-dimensional $L$-vector space

$$
D_{L}=D \otimes_{K_{0}^{\prime} \otimes_{\mathbb{Q} p} L} L,
$$

with the induced $N$ coming from $B_{\text {st }}$, and with $r: W_{K} \rightarrow \operatorname{GL}\left(D_{L}\right)$ defined by $r(w)=\phi^{-d(w)} \circ \bar{w}$. Here $\bar{w}$ denotes the image of $w$ in $\operatorname{Gal}\left(K^{\prime} \mid K\right)$, and $d(w)$ gives the power of arithmetic Frobenius which $w$ induces. The ensuing Weil-Deligne representation becomes unramified upon restriction to $W_{K^{\prime}}$. We will denote it by $\operatorname{WD}(\rho)=\left(r, N, D_{L}\right)$ throughout the text.

The Breuil-Schneider conjecture asks for a characterization of the data arising in this fashion, assuming all Hodge-Tate numbers are distinct. To state it, start with abstract data. Firstly, for each embedding $\tau: K \hookrightarrow L$, say we are given $n$ distinct integers $H T_{\tau}=\left\{i_{1, \tau}<\cdots<i_{n, \tau}\right\}$. Secondly, say we are given some 
$n$-dimensional Weil-Deligne representation WD, with coefficients in $L$, which becomes unramified after restriction to $W_{K^{\prime}}$. With this data, we will associate a locally algebraic representation $\mathrm{BS}$ of $\mathrm{GL}_{n}(K)$, with coefficients in $L$. The algebraic part is defined in terms of the $H T_{\tau}$, the smooth part in terms of WD. Our data should come from a Galois representation $\rho$, as above, precisely when BS has a $\mathrm{GL}_{n}(K)$-stable $\mathcal{O}_{L}$-lattice, the unit ball of an invariant norm.

The following was also announced as Conjecture 4.1 in Breuil's [2010] ICM address:

The Breuil-Schneider conjecture [2007, Conjecture 4.3]. The following are equivalent:

(1) The data $H T_{\tau}$ and $\mathrm{WD}^{\mathrm{F}-\mathrm{ss}}$ arise from a potentially semistable $\rho$.

(2) BS admits a norm $\|\cdot\|$, invariant under the action of $\operatorname{GL}_{n}(K)$.

Before we recall the status of the conjecture, we return to the definition of BS.

- Algebraic part. Introduce $b_{j, \tau}=-i_{n+1-j, \tau}-(j-1)$. That is, write the $i_{j, \tau}$ in the opposite order, change signs, and subtract $(0,1, \ldots, n-1)$. We let $\xi_{\tau}$ be the irreducible algebraic $L$-representation of $\mathrm{GL}_{n}$, of highest weight

$$
b_{1, \tau} \leq b_{2, \tau} \leq \cdots \leq b_{n, \tau}
$$

relative to the lower triangular Borel. Their tensor product $\xi=\bigotimes_{\tau} \xi_{\tau}$, with $\tau$ running over $\operatorname{Hom}(K, L)$, is then an irreducible algebraic representation of $\mathrm{GL}_{n}\left(K \otimes_{\mathbb{Q}_{p}} L\right)$ over $L$. We will view $\xi$ as a representation of $\mathrm{GL}_{n}(K)$.

- Smooth part. By the classical local Langlands correspondence [Harris and Taylor 2001], the Frobenius semisimplification $\mathrm{WD}^{\mathrm{F}-\mathrm{ss}}$ is isomorphic to $\operatorname{rec}_{n}\left(\pi^{\circ}\right)$ for some irreducible admissible smooth representation $\pi^{\circ}$ of $\mathrm{GL}_{n}(K)$, defined

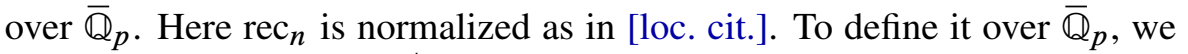
need to fix a square root $q^{1 / 2}$, where $q=\# \mathbb{F}_{K}$. By the Langlands classification, one has

$$
\operatorname{Ind}_{P}\left(Q\left(\Delta_{1}\right) \otimes \cdots \otimes Q\left(\Delta_{r}\right)\right) \stackrel{!}{\longrightarrow} \pi^{\circ},
$$

a unique irreducible quotient, where the $Q\left(\Delta_{i}\right)$ are generalized Steinberg representation built from the $\Delta_{i}$, which are segments of supercuspidals, suitably ordered. The smooth part of BS is now defined to be

$$
\pi=\operatorname{Ind}_{P}\left(Q\left(\Delta_{1}\right) \otimes \cdots \otimes Q\left(\Delta_{r}\right)\right) \otimes|\operatorname{det}|^{(1-n) / 2},
$$

or rather its model over $L$, which is independent of the choice of $q^{1 / 2}$. Note that $\pi \simeq \pi^{\circ} \otimes|\operatorname{det}|^{(1-n) / 2}$ if and only if $\pi^{\circ}$ is generic (that is, has a Whittaker model). For that reason, the association $\mathrm{WD} \mapsto \pi$ is often called the generic local Langlands correspondence. 
We let BS $=\xi \otimes_{L} \pi$, following [Breuil and Schneider 2007] (although they do not use the notation BS). In fact, we will find it more convenient to work with a different normalization. In the above construction there is a choice of a sign, essentially reflected in whether one twists by $|\operatorname{det}|^{(1-n) / 2}$ or its inverse. The latter is more commonly used in the references we rely on. The resulting representation is just a twist of BS by a harmless explicit $p$-adically unitary continuous character. Namely,

$$
\widetilde{\mathrm{BS}}=\mathrm{BS} \otimes_{L} \mu^{n-1}, \quad \mu(g)=N_{K \mid \mathbb{Q}_{p}}(\operatorname{det} g)^{\times},
$$

where $a^{\times}=a|a|_{p}=\operatorname{BS}\left(\chi_{\text {cyc }}\right)(a) \in \mathbb{Z}_{p}^{\times}$denotes the unit factor of an $a \in \mathbb{Q}_{p}^{*}$. Of course, $\widetilde{\mathrm{BS}}$ has an invariant norm if and only if BS does, so it makes no real difference. It reflects a Tate twist: $\widetilde{\mathrm{BS}}(\rho)$ is nothing but $\mathrm{BS}\left(\rho \otimes \chi_{\text {cyc }}^{n-1}\right)$.

The implication $(2) \Rightarrow(1)$ in the conjecture is in fact completely known. After many cases were worked out in [Schneider and Teitelbaum 2006; Breuil and Schneider 2007], the general case was settled by Y. Hu in his thesis [2009]. In fact, Hu proves that (1) is equivalent to the Emerton condition, which is a purely group-theoretical condition:

$$
J_{P}(\mathrm{BS})^{Z_{M}^{+}=\chi} \neq 0 \quad \Longrightarrow \quad \forall z \in Z_{M}^{+}:\left|\delta_{P}^{-1}(z) \chi(z)\right|_{p} \leq 1 .
$$

Here $J_{P}$ is Emerton's generalization of the Jacquet functor [2006a; 2007]. The heart of Hu's proof is to translate (3) into finitely many inequalities relating the Hodge polygon to the Newton polygon. In the vein of [Fontaine and Rapoport 2005], he is then able to show the existence of an admissible filtration compatible with the given data. The implication $(2) \Rightarrow(3)$ is relatively easy.

What remains is to produce an invariant norm on $\mathrm{BS}(\rho)$, for any potentially semistable $\rho$ (with distinct Hodge-Tate weights). One of the main motivations for writing this paper was to make progress in this direction, (1) $\Rightarrow(2)$. The supercuspidal case was dealt with in [Breuil and Schneider 2007] by purely local methods (see Theorem 5.2 in [loc. cit.]). The desired norm can be found either by compact induction, or by looking at matrix coefficients.

When $\mathrm{WD}(\rho)$ is indecomposable (in other words, $\pi^{\circ}=Q(\Delta)$ is generalized Steinberg), we proved (1) $\Rightarrow$ (2) in [Sorensen 2013]. Here (as in the supercuspidal case) the Emerton condition boils down to just integrality of the central character, and in fact the resulting conjecture was stated explicitly as Conjecture 5.5 in [Breuil and Schneider 2007]. The key point of [Sorensen 2013] was to make use of the fact that $Q(\Delta)$ is a discrete series representation, and therefore admits a pseudocoefficient. Inserting this as a test function in the trace formula for a certain definite unitary group, one can pass to a global setup (à la Grunwald-Wang). Finally, the desired norm was found by relating classical algebraic modular forms to the completed cohomology for the definite unitary group, $\widetilde{H}^{0}$. (This is within the framework of [Emerton 2006b], in which completed cohomology was defined and studied extensively for the 
tower of locally symmetric spaces - for any reductive group. In our case, however, these constructions boil down to just continuous functions on profinite sets, and Emerton's general machinery is not needed.) The argument in [Sorensen 2013] is purely group-theoretical, and in fact carries over to any connected reductive group over $\mathbb{Q}_{p}$, exploiting a compact form (using a Galois cohomological computation of Borel and Harder, which shows the existence of locally prescribed forms).

The purpose of this paper is to prove results which complement those of [Sorensen 2013]. The idea of relating algebraic modular forms to $\widetilde{H}^{0}$, already present in [Emerton 2006b], can be pushed further, now that local-global compatibility at $p=\ell$ is available in the "book project" context. This was proved recently by Barnet-Lamb, Gee, Geraghty, and Taylor in the so-called Shin-regular case [BarnetLamb et al. 2014], and this regularity hypothesis was then shown to be unnecessary by Caraiani, as part of her Harvard Ph.D. thesis - see [Caraiani 2012]. This results in the following somewhat vague Theorem A, which we will make more precise in Theorem B below.

Theorem A. The Breuil-Schneider conjecture holds for representations $\rho$ that come from stable automorphic representations $\pi$ on definite unitary groups, of regular weight (that is, restrictions $\rho \simeq r_{\pi, \iota} \mid \Gamma_{\mathcal{K}_{w}}$, at places $w \mid p$, of irreducible automorphic Galois representations $r_{\pi, \iota}$ of regular weight).

Eigenvarieties. We will combine the approaches of [Chenevier 2009; Emerton 2006b]. Thus let $\mathcal{K}$ be a CM field, with maximal totally real subfield $F$. Let $D$ be a central simple $\mathcal{K}$-algebra of $\operatorname{dim}_{\mathcal{K}}(D)=n^{2}$, equipped with an anti-involution $\star$ of the second kind (that is, $\left.\star\right|_{\mathcal{K}}$ is conjugation). We introduce the unitary $F$-group $U=U(D, \star)$, an outer form of $\operatorname{GL}(n)$, which becomes the inner form $D^{\times}$over $\mathcal{K}$. It will be convenient to also introduce $G=\operatorname{Res}_{F \mid \mathbb{Q}}(U)$. We will always assume that $U$ is a totally definite group. In other words, we assume that $G(\mathbb{R})$ is a compact Lie group, which is therefore a product of copies of $U(n)$.

We will fix a prime number $p$ such that every place $v \mid p$ of $F$ splits in $\mathcal{K}$, and such that $D_{w}^{\times} \simeq \operatorname{GL}_{n}\left(\mathcal{K}_{w}\right)$ for every $w \mid v$. To be safe, we fix an isomorphism $D_{w} \stackrel{\sim}{\longrightarrow} M_{n}\left(\mathcal{K}_{w}\right)$ once and for all (uniquely determined up to inner automorphisms). To keep track of various identifications, it is customary to choose a place $\tilde{v}$ of $\mathcal{K}$ above every $v \mid p$. Once and for all, we also choose an isomorphism $\iota: \mathbb{C} \stackrel{\sim}{\longrightarrow} \overline{\mathbb{Q}}_{p}$. This gives rise to an identification

$$
\operatorname{Hom}(F, \mathbb{R})=\operatorname{Hom}(F, \mathbb{C}) \simeq \operatorname{Hom}\left(F, \overline{\mathbb{Q}}_{p}\right)=\bigsqcup_{v \mid p} \operatorname{Hom}\left(F_{v}, \overline{\mathbb{Q}}_{p}\right),
$$

and similarly for $\operatorname{Hom}(\mathcal{K}, \mathbb{C})$. By assumption $F_{v} \simeq \mathcal{K}_{w}$ for $w \mid v$, so the choices $\{\tilde{v}\}$ just amount to fixing a CM-type $\Phi$, which is ordinary for $\iota$, in the sense of [Katz 1978]. This will ensure that the various identifications we make are compatible. 
The eigenvariety for $G$ depends on the choice of a tame level $K^{p} \subset G\left(\mathbb{A}_{f}^{p}\right)$. It is a reduced rigid analytic space $\mathbb{X}_{/ E}$, where we take $E$ to be the Galois closure of $F$ in $\overline{\mathbb{Q}}_{p}$, with additional structure:

$$
\chi: \mathbb{X} \rightarrow \widehat{T}, \quad \lambda: \mathcal{H}\left(K^{p}\right)^{\mathrm{sph}} \rightarrow \mathcal{O}(\mathbb{X}) .
$$

Here $\widehat{T}_{/ E}$ is weight space, parametrizing locally analytic characters of $T\left(\mathbb{Q}_{p}\right)$, and $\mathcal{H}\left(K^{p}\right)^{\mathrm{sph}}$ is the spherical central subalgebra of the Hecke $E$-algebra $\mathcal{H}\left(K^{p}\right)$. Finally, $\mathcal{O}(\mathbb{X})$ is the Banach algebra of rigid functions on $\mathbb{X}$. Moreover, there is a Zariski-dense subset $X_{\mathrm{cl}} \subset \mathbb{X}\left(\overline{\mathbb{Q}}_{p}\right)$ such that the evaluation

$$
\mathbb{X}\left(\overline{\mathbb{Q}}_{p}\right) \longrightarrow\left(\widehat{T} \times \operatorname{Spec} \mathcal{H}\left(K^{p}\right)^{\mathrm{sph}}\right)\left(\overline{\mathbb{Q}}_{p}\right), \quad x \mapsto\left(\chi_{x}, \lambda_{x}\right),
$$

identifies $X_{\mathrm{cl}}$ with the set of classical points: roughly this means that, first of all, $\chi_{x}=\psi_{x} \cdot \theta_{x}$ is locally algebraic ( $\psi_{x}$ is the algebraic part, $\theta_{x}$ is the smooth part), and there exists an automorphic representation $\pi$ of weight $\psi_{x}$ such that $\pi_{p} \hookrightarrow \operatorname{Ind}_{B}^{G}\left(\theta_{x}\right)$, and $\mathcal{H}\left(K^{p}\right)^{\mathrm{sph}}$ acts on $\pi_{f}^{K^{p}} \neq 0$ by the character $\lambda_{x}$. (The condition that $\pi_{p}$ embeds in a principal series is the analogue of the "finite slope" requirement showing up in the classical works of Coleman, Mazur and others. The choice of a $\theta_{x}$ is called a refinement of $\pi$.) Thus a classical point $x$ carries a little more information than just an automorphic representation $\pi_{x}$; it keeps track of the refinement $\theta_{x}$. We refer to Section 4 below for more details on eigenvarieties, which contains references to their various constructions.

It is of utmost importance to us that the eigenvariety carries a family of Galois representations. To be more precise, if we let $\Sigma=\Sigma\left(K^{p}\right)$ be the set of ramified places, there is a unique continuous $n$-dimensional pseudorepresentation into the unit ball $\mathcal{O}(\mathbb{X})^{\leq 1}$ of $\mathcal{O}(\mathbb{X})$, say

$$
\mathcal{T}: \Gamma_{\mathcal{K}, \Sigma} \rightarrow \mathcal{O}(\mathbb{X})^{\leq 1}
$$

associated with $\lambda: \mathcal{H}\left(K^{p}\right)^{\mathrm{sph}} \rightarrow \mathcal{O}(\mathbb{X})$, in the sense that for all places $w \notin \Sigma$,

$$
\mathcal{T}\left(\operatorname{Frob}_{w}\right)=\lambda\left(b_{w \mid v}\left(h_{w}\right)\right) .
$$

Here $h_{w}$ is the element of the spherical Hecke algebra for $\operatorname{GL}_{n}\left(\mathcal{K}_{w}\right)$, which acts via the sum of the (integral) Satake parameters on spherical vectors, and

$$
b_{w \mid v}: \mathcal{H}\left(\mathrm{GL}_{n}\left(\mathcal{K}_{w}\right), \mathrm{GL}_{n}\left(\mathcal{O}_{\mathcal{K}_{w}}\right)\right) \longrightarrow \mathcal{H}\left(U\left(F_{v}\right), K_{v}\right)
$$

is the standard base change homomorphism between the pertaining spherical Hecke algebras, see (4.2) on p. 17 in [Mínguez 2011], and his Theorem 4.1. Here $K_{v}$ is the factor of $K^{p}$ at $v$. It is a hyperspecial maximal compact subgroup of $U\left(F_{v}\right)$ (note that this does not determine $K_{v}$ up to conjugation when $n$ is even, but our $K^{p}$ is fixed from the outset). In particular, for each $x \in \mathbb{X}\left(\overline{\mathbb{Q}}_{p}\right)$ there is a unique 
semisimple Galois representation

$$
r_{x}: \Gamma_{\mathcal{K}, \Sigma} \rightarrow \mathrm{GL}_{n}\left(\overline{\mathbb{Q}}_{p}\right), \quad \mathcal{T}_{x}=\operatorname{tr}\left(r_{x}\right) .
$$

In fact, the way $\mathcal{T}$ is constructed is by first defining $r_{x}$ for regular classical points $x \in X_{\mathrm{cl}}$, by which we mean the dominant character $\psi_{x}$ is given by a strictly decreasing sequence of integers (at some place). We will denote this subset of points by $X_{\text {reg }} \subset X_{\mathrm{cl}}$. Thanks to [White 2012, Theorem 6.1, p. 28] (now superseded by [Kaletha et al. 2014]) this guarantees that $\pi$ has a base change to $\mathrm{GL}_{n}\left(\mathbb{A}_{\mathcal{K}}\right)$ of the form $\Pi=\boxplus \Pi_{i}$, where the $\Pi_{i}$ are cohomological cuspidal (as opposed to just discrete) automorphic representations to which one can attach Galois representations. Now, $X_{\text {reg }}$ can be shown to be Zariski dense, and a formal argument in [Chenevier 2004] interpolates the pseudocharacters $\operatorname{tr}\left(\rho_{x}\right)$ for $x \in X_{\text {reg }}$ by a unique $\mathcal{T}$, which one can then specialize at any point $x \in \mathbb{X}\left(\overline{\mathbb{Q}}_{p}\right)$.

We can now rephrase the statement in Theorem A in terms of eigenvarieties: if $x \in X_{\text {reg }}$ is a (classical) point such that $r_{x}$ is irreducible (as a representation of $\Gamma_{\mathcal{K}}$ ), and $w \mid p$ is a place of $\mathcal{K}$, then $\left.r_{x}\right|_{\Gamma_{\mathcal{K}}}$ is potentially semistable, and its locally algebraic representation $\mathrm{BS}\left(\left.r_{x}\right|_{\Gamma_{\mathcal{K}}}\right)$ admits a $\mathrm{GL}_{n}\left(\mathcal{K}_{w}\right)$-invariant norm.

Our main result. The actual construction of an invariant norm $\|\cdot\|$ is more interesting than its mere existence. It comes out of a much more precise result, which we now describe. Fix a finite extension $L \mid E$. At each point $x \in \mathbb{X}(L)$, we have a pseudorepresentation $\mathcal{T}_{x}: \Gamma_{\mathcal{K}, \Sigma} \rightarrow L$ (the trace of an actual representation $\rho_{x}$, which may or may not be defined over $L$ ). On the other hand, to $x \in \mathbb{X}(L)$ we associate the Banach $L$-space

$$
\mathcal{B}_{x}=\left(L \otimes_{E} \tilde{H}^{0}\left(K^{p}\right)\right)^{\mathfrak{h}=\lambda_{x}},
$$

where $\mathfrak{h}=\mathcal{H}\left(K^{p}\right)^{\mathrm{sph}}$ is shorthand notation. This space is really very concrete. The completed cohomology $\widetilde{H}^{0}\left(K^{p}\right)$ is here nothing but the space of all continuous functions

$$
f: Y\left(K^{p}\right) \rightarrow E, \quad Y\left(K^{p}\right)=\lim _{K_{p}} Y\left(K_{p} K^{P}\right), \quad Y(K)=G(\mathbb{Q}) \backslash G\left(\mathbb{A}_{f}\right) / K
$$

with supremum norm. The superscript $\mathfrak{h}=\lambda_{x}$ means we take the eigenspace for the character $\lambda_{x}: \mathfrak{h} \rightarrow L$ (not the generalized eigenspace). Note that $\mathcal{B}_{x}$ is much more than just a Banach $L$-space: For one thing, it is a Banach module for the Banach-Hecke algebra $\hat{\mathcal{H}}\left(K^{p}\right)$ (see [Schneider and Teitelbaum 2006] for a detailed discussion of these). For another thing, there is a natural $\widehat{\mathcal{H}}\left(K^{p}\right)$-linear action of $G\left(\mathbb{Q}_{p}\right)$ by isometries of $\mathcal{B}_{x}$, which is admissible (meaning that its mod $p$ reduction $\overline{\mathcal{B}}_{x}$ is a smooth admissible representation of $G\left(\mathbb{Q}_{p}\right)$ over $\mathbb{F}_{L}$, in the usual sense). Now, for two points $x, x^{\prime} \in \mathbb{X}(L)$,

$$
\mathcal{T}_{x}=\mathcal{T}_{x^{\prime}} \Longleftrightarrow \lambda_{x}=\lambda_{x^{\prime}} \Longleftrightarrow \mathcal{B}_{x}=\mathcal{B}_{x^{\prime}},
$$


since each $b_{w \mid v}$ is onto; see Corollary 4.2 in [Mínguez 2011] (a fact also used on p. 10 of [Clozel et al. 2011]). In other words, the set of all pairs $\left(\mathcal{T}_{x}, \mathcal{B}_{x}\right)$ is the graph of a bijection between the set of pseudorepresentations $\left\{\mathcal{T}_{x}: x \in \mathbb{X}(L)\right\}$, and the set of Banach representations $\left\{\mathcal{B}_{x}: x \in \mathbb{X}(L)\right\}$. That is, there is a one-to-one correspondence:

$$
\left\{\begin{array}{c}
n \text {-dimensional pseudo- } \\
\text { representations } \mathcal{T}: \Gamma_{\mathcal{K}, \Sigma} \rightarrow L \\
\text { coming from } \mathbb{X}(L)
\end{array}\right\} \longleftrightarrow\left\{\begin{array}{c}
\text { Banach } \hat{\mathcal{H}}_{L}\left(K^{p}\right) \text {-modules } \mathcal{B} \\
\text { with admissible unitary } \\
G\left(\mathbb{Q}_{p}\right) \text {-action, coming from } \mathbb{X}(L)
\end{array}\right\} .
$$

Here $\mathcal{T} \leftrightarrow \mathcal{B}$ means there is a point $x \in \mathbb{X}(L)$ such that $\mathcal{T}=\mathcal{T}_{x}$ and $\mathcal{B}=\mathcal{B}_{x}$. (We say that a pseudocharacter $\mathcal{T}: \Gamma_{\mathcal{K}, \Sigma} \rightarrow L$ comes from $\mathbb{X}(L)$ if it is of the form $\mathcal{T}_{x}$ for a point $x \in \mathbb{X}(L)$, and similarly for Banach modules.)

To ease the exposition, let us assume we have split ramification. That is, $S\left(K^{p}\right) \subset \operatorname{Spl}_{\mathcal{K} \mid F}$. Then local base change is defined everywhere, and there is a unique automorphic representation $\pi_{x}$ associated with a point $x \in X_{\mathrm{cl}}$ such that $r_{x}$ is irreducible (indeed its global base change is cuspidal and determined almost everywhere). Experts have informed us that $m\left(\pi_{x}\right)=1$, but we have not been able to locate it in the literature. Our main result in this paper is the following, which will be proved in Section 6 .

Theorem B. Assume $S\left(K^{p}\right) \subset \operatorname{Spl}_{\mathcal{K} \mid F}$. For each classical point $x \in X_{\text {reg }} \cap X_{\text {irr }}$, defined over $L$, such that $m\left(\pi_{x}\right)=1$, there is a unique (up to topological equivalence) Banach space $B\left(r_{x}\right)$ over $L$ with an admissible unitary $G\left(\mathbb{Q}_{p}\right)$-action such that:

(1) $B\left(r_{x}\right)^{\mathrm{ralg}} \simeq \widetilde{\mathrm{BS}}\left(r_{x}\right):=\bigotimes_{v \mid p} \widetilde{\mathrm{BS}}\left(\left.r_{x}\right|_{\Gamma_{\mathcal{K}_{\tilde{v}}}}\right)$ is dense in $B\left(r_{x}\right)$.

(2) There is a $G\left(\mathbb{Q}_{p}\right) \times \hat{\mathcal{H}}\left(K^{p}\right)$-equivariant topological isomorphism,

$$
B\left(r_{x}\right) \otimes\left(\bigotimes_{v \nmid p} \pi_{x, v}^{K_{v}}\right) \stackrel{\sim}{\longrightarrow} \overline{\mathcal{B}_{x}^{\text {ralg }}} .
$$

(Here $\overline{\mathcal{B}_{x}^{\text {ralg }}}$ denotes the closure of the locally regular-algebraic ${ }^{2}$ vectors in $\mathcal{B}_{x}$.)

(3) If $r_{x}$ is crystalline above $p$, there is a continuous $G\left(\mathbb{Q}_{p}\right)$-equivariant map with dense image,

$$
B_{\xi_{x}, \zeta_{x}} \longrightarrow B\left(r_{x}\right)
$$

which restricts to an isomorphism $H_{\xi_{x}, \zeta_{x}} \stackrel{\sim}{\longrightarrow} B\left(r_{x}\right)^{\mathrm{ralg}}$. (Here $H_{\xi, \zeta}$ and $B_{\xi, \zeta}$ are the spaces introduced by Schneider and Teitelbaum [2006], and we take $\xi_{x}$ of highest weight $\psi_{x}$, and $\zeta_{x}$ to be the eigensystem of $\theta_{x}$.)

${ }^{2}$ Apparently "regularity" is no longer an issue here (see Section 2.3 below). We warn the reader that the terminology "regular-algebraic" can be misleading here. It is stronger than "cohomological". It means the Hodge-Tate gaps (for some embedding $\tau$ ) are at least $t w o$. The condition arises in endoscopy when base-changing to $\mathrm{GL}_{n}$. 
Since Theorem B is exclusively about classical points, it can be formulated purely in terms of automorphic Galois representations (thus refining and extending Theorem A): for instance, it says that $\bigotimes_{v \mid p} \widetilde{\mathrm{BS}}\left(\left.r_{\pi, l}\right|_{\Gamma_{\mathcal{K}_{\tilde{v}}}}\right)$ admits a unitary Banach completion $B\left(r_{\pi, l}\right)$ such that

$$
B\left(r_{\pi, l}\right) \otimes\left(\pi_{f}^{p}\right)^{K^{p}} \stackrel{\sim}{\longrightarrow} \overline{\widetilde{H}^{0}\left(K^{p}\right)[\pi]^{\text {ralg }}} .
$$

The eigenvariety formulation of Theorem $\mathrm{B}$ is more amenable to generalization, and is meant to signal what we expect to be true. Namely, say $x \in \mathbb{X}(L)$ is a point which is not a priori known to be classical, but which behaves like a classical point - in that $r_{x} \mid \Gamma_{\mathcal{K}_{w}}$ is potentially semistable of regular weight, for all $w \mid p$. Then Theorem B should hold verbatim for $x$, which essentially means $x$ is necessarily classical (cf. the Fontaine-Mazur conjecture). Furthermore, if a $p$-adic local Langlands correspondence exists in this generality, we believe that $B\left(r_{x}\right)=\hat{\bigotimes}_{v \mid p} B\left(\left.r_{x}\right|_{\Gamma_{\mathcal{K}_{\tilde{v}}}}\right)$ should satisfy something along the lines of (2), for any point $x$ : the representations $\pi_{x, v}$ are still defined for $v \nmid p$, via classical local Langlands, and we would hope that $B\left(r_{x}\right) \otimes\left(\otimes_{v \nmid p} \pi_{x, v}^{K_{v}}\right)$ at least embeds into $\mathcal{B}_{x}$ (perhaps assuming the local restrictions $\left.r_{x}\right|_{\Gamma_{\mathcal{K}_{\tilde{v}}}}$ are irreducible for $v \mid p$ ).

Caraiani, Emerton, Gee, Geraghty, Paskunas, and Shin [Caraiani et al. 2014] have recently announced spectacular work in the principal series case. They employ a delicate variant of the Taylor-Wiles-Kisin patching method (allowing the weight and $p$-level to vary freely), and construct a candidate for the $p$-adic local Langlands correspondence for $\mathrm{GL}_{n}(F)$ via deformations of Galois representations. This makes use of auxiliary global data, and they are unable to show the proposed candidate only depends on the local data at $p$. However, they are able to say enough about their construction to reduce the Breuil-Schneider conjecture to folklore conjectures in the automorphy lifting world. For instance, they prove that $\mathrm{BS}(\rho)$ admits an admissible unitary Banach completion for potentially crystalline $\rho: \Gamma_{F} \rightarrow \mathrm{GL}_{n}(E)$ which lie on an automorphic component of a certain potentially crystalline deformation ring - which is expected to always hold. Morally, we show Breuil-Schneider for $\rho=\left.r_{\pi, l}\right|_{\Gamma_{F w}}$, and [Caraiani et al. 2014] shows it for potentially crystalline lifts $\rho$ of $\bar{r}_{\pi, l} \mid \Gamma_{F w}$ - which of course is more general, but their techniques rely on heavy machinery.

Organization of the paper. Section 2 sets up notation used throughout the paper, and recalls how to attach Galois representations to automorphic forms on definite unitary groups. We work out the relation between Breuil-Schneider's normalization BS and our preferred normalization $\widetilde{\mathrm{BS}}$, which is what occurs naturally in cohomology. In Section 3 we briefly discuss completed cohomology in this context, which boils down to just $\widetilde{H}^{0}$ — continuous functions on a profinite set, and relate its locally algebraic vectors to algebraic modular forms. This is crucial for the general 
strategy of our paper (and its prequel [Sorensen 2013]). At the end we prove Theorem A. Section 4 introduces eigenvarieties $\mathbb{X}$ and the (semisimple) Galois representations $\rho_{x}$ they carry - fundamental notions in Section 5, which discusses the Banach representations $\mathcal{B}_{x}$ associated with arbitrary points $x$ on $\mathbb{X}$, and sets up the bijection $\rho_{x} \leftrightarrow \mathcal{B}_{x}$ in Theorem B. Section 6 goes into detail about how one can naturally complete $\widetilde{\mathrm{BS}}$ and get a "rough" candidate for a $p$-adic local Langlands correspondence $B\left(r_{x}\right) \hookrightarrow \mathcal{B}_{x}$ when $x$ is classical, and explains to what it extent it satisfies local-global compatibility. We specialize to the crystalline case, and show that our candidate $B\left(r_{x}\right)$ is (almost) a quotient of the purely locally defined Banach representation $B_{\xi_{x}}, \zeta_{x}$ of Schneider and Teitelbaum - as predicted. Section 7 is logically independent of the rest of the paper, and we include it here only for future reference. It gives an in-depth treatment of "Zariski density of crystalline points" in this context, by expanding on an argument of Emerton (building on work of Katz) in the case of GL(2)/QQ . Density should be important in future work on extending parts of Theorem B to (a priori) nonclassical points.

\section{Automorphic Galois representations}

We start out by summarizing what is currently known about attaching Galois representations to automorphic representations of definite unitary groups. Due to the work of many people, we now have an almost complete understanding of this, and below we merely navigate the existing literature. We claim no originality in this section. Our goal is simply to state the precise result. Particularly, we want to emphasize the local-global compatibility at $p=\ell$, recently proved in [Barnet-Lamb et al. 2014; Caraiani 2012], which is fundamental for this paper.

2.1. Definite unitary groups. Throughout this article, we fix a totally real field $F$, and a $\mathrm{CM}$ extension $\mathcal{K}$. We let $c$ denote the nontrivial element of $\operatorname{Gal}(\mathcal{K} \mid F)$. The places of $F$ will usually be denoted by $v$, and those of $\mathcal{K}$ by $w$. We are interested in outer forms $U$ of $\operatorname{GL}(n)_{F}$, which become an inner form $D^{\times}$over $\mathcal{K}$. Here $D$ is a central simple $\mathcal{K}$-algebra, of $\operatorname{dim}_{\mathcal{K}}(D)=n^{2}$. These forms are unitary groups $U=U(D, \star)$, where $\star$ is an anti-involution on $D$ of the second kind $\left(\left.\star\right|_{\mathcal{K}}=c\right)$. Thus, for any $F$-algebra $R$,

$$
U(R)=\left\{x \in\left(D \otimes_{F} R\right)^{\times}: x x^{\star}=1\right\} .
$$

We will always assume from now on that $U\left(F \otimes_{\mathbb{Q}} \mathbb{R}\right)$ is compact. Thus, by making a choice of a CM-type $\Phi$, the group may be identified with $U(n)^{\operatorname{Hom}(F, \mathbb{R})}$ (up to conjugation). It will be convenient to work over the rationals, and introduce $G=\operatorname{Res}_{F \mid \mathbb{Q}}(U)$. With the same $\Phi$ one identifies $G(\mathbb{C})$ with $\mathrm{GL}_{n}(\mathbb{C})^{\operatorname{Hom}(F, \mathbb{R})}$.

2.2. Weights of automorphic representations. Following standard notation in the subject, $\left(\mathbb{Z}^{n}\right)_{+}^{\operatorname{Hom}(\mathcal{K}, \mathbb{C})}$ will denote the set of tuples $a=\left(a_{\tau}\right)_{\tau \in \operatorname{Hom}(\mathcal{K}, \mathbb{C})}$, where each 
$a_{\tau}=\left(a_{\tau, j}\right)$ itself is a decreasing tuple,

$$
a_{\tau}=\left(a_{\tau, 1} \geq a_{\tau, 2} \geq \cdots \geq a_{\tau, n}\right),
$$

of integers. In the obvious way, we can identify $a_{\tau}$ with a dominant weight for $\mathrm{GL}(n)$, relative to the upper triangular Borel. We say $a_{\tau}$ is regular if all the inequalities above are strict. We say $a$ is regular if $a_{\tau}$ is regular for some $\tau$.

Now, let $\pi=\pi_{\infty} \otimes \pi_{f}$ be an automorphic representation of $U\left(\mathbb{A}_{F}\right)$. We will define what it means for $\pi$ to have weight $a$ : Every embedding $\tau: \mathcal{K} \hookrightarrow \mathbb{C}$ restricts to a $\sigma: F \hookrightarrow \mathbb{R}$, which corresponds to an infinite place $v=v(\sigma)$ of $F$. With this notation, $\tau$ identifies $U\left(F_{v}\right) \simeq U(n)$, under which $\pi_{v}$ should be equivalent to the contragredient $\breve{V}_{a_{\tau}}$, or rather its restriction. Here $V_{a_{\tau}}$ is the irreducible algebraic representation of $\mathrm{GL}_{n}(\mathbb{C})$ of highest weight $a_{\tau}$.

Remark. We must have $V_{a_{\tau c}}=\breve{V}_{a_{\tau}}$. In other words, $a_{\tau c, j}=-a_{\tau, n+1-j}$.

2.3. Associating Galois representations. We have introduced enough notation in order to formulate the following main result, the foundation for our work. As mentioned already, this is the culmination of collaborative efforts of a huge group of outstanding mathematicians, as will become clear below.

Theorem 1. Choose a prime $p$, and an isomorphism $\iota: \mathbb{C} \stackrel{\sim}{\longrightarrow} \overline{\mathbb{Q}}_{p}$. Let $\pi$ be an automorphic representation of $U\left(\mathbb{A}_{F}\right)$ such that $\pi_{\infty}$ has regular weight $a$. Then there exists a unique continuous semisimple Galois representation

$$
\rho_{\pi, l}: \Gamma_{\mathcal{K}}=\operatorname{Gal}(\overline{\mathbb{Q}} \mid \mathcal{K}) \rightarrow \mathrm{GL}_{n}\left(\overline{\mathbb{Q}}_{p}\right)
$$

such that the following properties are satisfied:

(a) $\breve{\rho}_{\pi, \iota} \simeq \rho_{\pi, \iota}^{c} \otimes \epsilon_{\mathrm{cyc}}^{n-1}$,

(b) For every finite place $v$, and every $w \mid v$ (even those above $p$ ),

$$
\mathrm{WD}\left(\left.\rho_{\pi, \iota}\right|_{\Gamma_{\mathcal{K}}}\right)^{\mathrm{F}-\mathrm{ss}} \simeq \iota \operatorname{rec}\left(\mathrm{BC}_{w \mid v}\left(\pi_{v}\right) \otimes|\operatorname{det}|_{w}^{(1-n) / 2}\right)
$$

whenever $\mathrm{BC}_{w \mid v}\left(\pi_{v}\right)$ is defined, namely if $\pi_{v}$ is unramified or $v=w w^{c}$ splits.

(c) $\left.\rho_{\pi, \iota}\right|_{\Gamma_{\mathcal{K}_{w}}}$ is potentially semistable for all $w \mid p$, with Hodge-Tate numbers

$$
H T_{\iota \tau}\left(\left.\rho_{\pi, \iota}\right|_{\Gamma_{\mathcal{K}}}\right)=\left\{a_{\tau, j}+(n-j): j=1, \ldots, n\right\}
$$

for every $\tau: \mathcal{K} \hookrightarrow \mathbb{C}$ such that $\iota \tau$ lies above $w$. A word about our normalization here: $\rho_{\pi, \iota} \otimes_{\iota \tau, \mathcal{K}_{w}} \mathbb{C}_{\mathcal{K}_{w}}(i)$ has no $\Gamma_{\mathcal{K}_{w}}$-invariants unless $i$ is of the above form, in which case they form a line. Thus $H T_{\iota \tau}\left(\epsilon_{\mathrm{cyc}}\right)=\{-1\}$.

Proof. Ngo's proof of the fundamental lemma makes endoscopic transfer widely available. In particular, weak base change from any unitary group associated with $\mathcal{K} \mid F$ to $\mathrm{GL}_{n}\left(\mathbb{A}_{\mathcal{K}}\right)$ has matured. Building on work of Clozel and Labesse, 
White [2012] worked out the cohomological case completely. In our given setup, $\pi_{v}$ is automatically discrete series for all $v \mid \infty$, in which case Theorem 6.1 in [White 2012] — or rather the pertaining remarks 6.2 and 6.3 - yields an automorphic representation

$$
\Pi=\Pi_{1} \boxplus \cdots \boxplus \Pi_{t}
$$

of $\mathrm{GL}_{n}\left(\mathbb{A}_{\mathcal{K}}\right)$ which is an isobaric sum of mutually nonisomorphic conjugate selfdual cuspidal automorphic representations $\Pi_{i}$ of some $\mathrm{GL}_{n_{i}}\left(\mathbb{A}_{\mathcal{K}}\right)$ such that

$$
\Pi_{w}=\mathrm{BC}_{w \mid v}\left(\pi_{v}\right)
$$

for all $w \mid v$, where $v$ is split or archimedean, or $\pi_{v}$ is unramified. The regularity of $\pi_{\infty}$ ensures that the $\Pi_{i}$ are cuspidal (as opposed to just discrete), which in turn implies the previous equality at the archimedean places $w \mid v$. Let us spell it out in that case: Fix an embedding $\tau: \mathcal{K} \hookrightarrow \mathbb{C}$ inducing $\mathcal{K}_{w} \simeq \mathbb{C}$. Then,

$$
\phi_{\Pi_{w}}: \mathbb{C}^{*}=W_{\mathbb{C}} \simeq W_{\mathcal{K}_{w}} \rightarrow \mathrm{GL}_{n}(\mathbb{C})
$$

maps

$$
z \mapsto\left(\begin{array}{lll}
(z / \bar{z})^{-h_{1}+(n-1) / 2} & & \\
& \ddots & \\
& & (z / \bar{z})^{-h_{n}+(n-1) / 2}
\end{array}\right),
$$

for certain $h_{j} \in \mathbb{Z}$, which are given in terms of the weight by $h_{j}=a_{\tau, j}+(n-j)$. This last formula is worked out in [Bergeron and Clozel 2005], for example (see their Proposition 5.3.1, p. 63, which gives the Langlands parameters of cohomological representations of $U(a, b))$. These $h_{j}$ are distinct, so each $\Pi_{i} \otimes|\operatorname{det}|_{\mathcal{K}}^{\left(n_{i}-n\right) / 2}$ is regular-algebraic, essentially conjugate self-dual, and cuspidal. By Theorem A of [Barnet-Lamb et al. 2014], and the references therein, we can associate a Galois representation $r_{\Pi_{i}, l}$ to it satisfying the properties analogous to (a)-(c). As a remark, in [loc. cit.] local-global compatibility at $p$ is proved assuming Shin regularity, which is much weaker than regularity. In any case, the Shin regularity assumption was removed by Caraiani [2012]. It is then straightforward to check that the representation

$$
\rho_{\pi, \iota}=r_{\Pi_{1}, \iota} \oplus \cdots \oplus r_{\Pi_{t}, \iota}
$$

has the desired properties. It is uniquely determined by (b), by Tchebotarev.

Remark. It appears within reach to extend the previous argument to the irregular case. By [White 2012], one still has a weak base change $\boxplus_{i=1}^{t} \Pi_{i}$, but the $\Pi_{i}$ are only discrete, not cuspidal. By Shapiro's lemma in $(\mathfrak{g}, K)$-cohomology, these $\Pi_{i}$ should still be cohomological (of Speh type). By the Moeglin-Waldspurger description of the discrete spectrum of $\operatorname{GL}\left(n_{i}\right)$, one can in turn express each $\Pi_{i}$ as an isobaric sum of cusp forms, with which one can associate Galois representations. 
After having consulted several experts in the field, we are quite optimistic about this line of argument, and that the ubiquitous regularity ${ }^{3}$ assumption (appearing throughout this paper) can safely be dropped. However, we have not made any serious attempt to work out the details. We are hopeful that the recent joint work of Kaletha, Mínguez, Shin, and White [Kaletha et al. 2014], which complements [Mok 2014], should provide the strengthenings of [White 2012] needed.

2.4. The Breuil-Schneider recipe. We attach to a potentially semistable representation $\rho: \Gamma_{\mathcal{K}_{w}} \rightarrow \mathrm{GL}_{n}\left(\overline{\mathbb{Q}}_{p}\right)$, with distinct Hodge-Tate numbers, a locally algebraic representation $\operatorname{BS}(\rho)$ of $\mathrm{GL}_{n}\left(\mathcal{K}_{w}\right)$ on a $\overline{\mathbb{Q}}_{p}$-vector space, as in the introduction. The Breuil-Schneider conjecture is the mere existence of an invariant norm on $\operatorname{BS}(\rho)$. Our first goal is to prove the conjecture for $\rho=\left.\rho_{\pi, l}\right|_{\Gamma_{\mathcal{K}_{w}}}$ for any place $w$ of $\mathcal{K}$ above $p$. We will achieve this below. For now, we will compute $\operatorname{BS}\left(\left.\rho_{\pi, \iota}\right|_{\Gamma_{\mathcal{K}_{w}}}\right)$ explicitly, by relating it to the classical local Langlands correspondence.

In fact, we prefer to use a slightly different normalization: There is a choice of a sign involved in the recipe on p. 16 in [Breuil and Schneider 2007]. Instead of twisting by $|\operatorname{det}|_{w}^{(1-n) / 2}$, we prefer to twist by $|\operatorname{det}|_{w}^{(n-1) / 2}$ to make it more compatible with the previous notation. Consequently, $\mathrm{BS}(\rho)$ becomes twisted by an integral character.

Definition 2. For $a \in \mathbb{Q}_{p}^{*}$ we let $a^{\times}=a|a|_{p}$ denote its unit part. We introduce

$$
\mu: \mathrm{GL}_{n}\left(\mathcal{K}_{w}\right) \stackrel{\operatorname{det}}{\longrightarrow} \mathcal{K}_{w}^{*} \stackrel{N_{\mathcal{K}_{w} \mid \mathbb{Q}_{p}}}{\longrightarrow} \mathbb{Q}_{p}^{*} \longrightarrow \mathbb{Q}_{p}^{*} / p^{\mathbb{Z}} \simeq \mathbb{Z}_{p}^{\times} .
$$

That is, $\mu(g)=N_{\mathcal{K}_{w} \mid \mathbb{Q}_{p}}(\operatorname{det} g)^{\times}$. We will normalize $\operatorname{BS}(\rho)$ as follows:

$$
\widetilde{\mathrm{BS}}(\rho):=\mathrm{BS}(\rho) \otimes_{\overline{\mathbb{Q}}_{p}} \mu^{n-1} .
$$

(Of course, this has an invariant norm precisely when $\mathrm{BS}(\rho)$ does.)

Lemma 3.

$$
\widetilde{\mathrm{BS}}(\rho)=\mathrm{BS}(\rho(n-1)) \text {. }
$$

Proof. Note that the character $a \mapsto a^{\times}$(which maps $p \mapsto 1$, and is the identity on $\mathbb{Z}_{p}^{\times}$) corresponds to the $p$-adic cyclotomic character $\chi_{\text {cyc }}: \Gamma_{\mathbb{Q}_{p}} \rightarrow \mathbb{Z}_{p}^{\times}$via local class field theory $\mathbb{Q}_{p}^{*} \rightarrow \Gamma_{\mathbb{Q}_{p}}^{\mathrm{ab}}$. For any $p$-adic field $K$, it follows that $\mathrm{BS}\left(\chi_{\text {cyc }}\right)$ is simply the character $a \mapsto N_{K \mid \mathbb{Q}_{p}}(a)^{\times}$. Consequently, $\widetilde{\mathrm{BS}}(\rho)=\mathrm{BS}\left(\rho \otimes \chi_{\mathrm{cyc}}^{n-1}\right)$.

We compute it in the automorphic case. Given the local-global compatibility results of [Barnet-Lamb et al. 2014] (generalized in [Caraiani 2012]), this is basically just "bookkeeping".

\footnotetext{
${ }^{3}$ We emphasize that the use of "regularity" here is admittedly poor terminology, and should not be confused with "cohomological". It is stronger, in that the gaps between the Hodge-Tate weights should be at least two. Thus dropping the regularity assumption does not signal that we can say anything for $\mathrm{GL}_{2}\left(\mathbb{Q}_{p}\right)$ about weight-one forms, for instance.
} 
Corollary 4. Let $\pi$ be an automorphic representation of $U\left(\mathbb{A}_{F}\right)$ of regular weight a. Assume $\rho_{\pi, l}$ is absolutely irreducible (as a representation of the full Galois group $\left.\Gamma_{\mathcal{K}}\right)$. Let $v \mid p$ be a place of $F$, either split in $\mathcal{K}$, or such that $\pi_{v}$ is unramified. Then, for any place $w \mid v$ of $\mathcal{K}$, we have

$$
\widetilde{\mathrm{BS}}\left(\rho_{\pi, l} \mid \Gamma_{\mathcal{K}_{w}}\right)=\left(\bigotimes_{\sigma: \mathcal{K}_{w} \hookrightarrow \overline{\mathbb{Q}}_{p}} \breve{V}_{a_{\iota-1} \sigma}\right) \otimes_{\overline{\mathbb{Q}}_{p}}\left(\mathrm{BC}_{w \mid v}\left(\pi_{v}\right) \otimes_{\mathbb{C}, \iota} \overline{\mathbb{Q}}_{p}\right) .
$$

(We abuse notation, and let $V_{a_{\tau}}$ denote the irreducible algebraic representation of $\mathrm{GL}_{n}\left(\overline{\mathbb{Q}}_{p}\right)$ of highest weight $a_{\tau}$, as opposed to the complex representation from earlier chapters.)

Proof. What is denoted $\pi^{\text {unit }}$ in [Breuil and Schneider 2007] equals, in our case, $\mathrm{BC}_{w \mid v}\left(\pi_{v}\right) \otimes|\operatorname{det}|_{w}^{(1-n) / 2}$ (more precisely, $\bigotimes_{\mathbb{C}, \iota} \overline{\mathbb{Q}}_{p}$ ). When it is generic, the smooth part of $\mathrm{BS}(\rho)$ is

$$
\pi^{\text {unit }} \otimes_{\overline{\mathbb{Q}}_{p}}|\operatorname{det}|_{w}^{(1-n) / 2}=\left(\mathrm{BC}_{w \mid v}\left(\pi_{v}\right) \otimes|\operatorname{det}|_{w}^{1-n}\right) \otimes_{\mathbb{C}, \iota} \overline{\mathbb{Q}}_{p} .
$$

In the nongeneric case, $\pi^{\text {unit }}$ has to be replaced by a certain parabolically induced representation. However, if we assume $\rho_{\pi, l}$ is (globally) irreducible, we see that $\Pi=\mathrm{BC}_{\mathcal{K} \mid F}(\pi)$ must be cuspidal, and in particular $\Pi_{w}$ is generic. The algebraic part of $\mathrm{BS}(\rho)$ is constructed out of the Hodge-Tate numbers: What is denoted $i_{j, \sigma}$ in [Breuil and Schneider 2007], for an embedding $\sigma: \mathcal{K}_{w} \hookrightarrow \overline{\mathbb{Q}}_{p}$, equals $a_{\tau, n+1-j}+(j-1)$ in our notation, where $\sigma=\iota \tau$. In (8) on p. 17 of [Breuil and Schneider 2007], the numbers become

$$
b_{\tau, j}:=-i_{n+1-j, \sigma}-(j-1)=-a_{\tau, j}-(n-1) .
$$

Breuil and Schneider's $\rho_{\sigma}$ is the irreducible algebraic representation of $\mathrm{GL}_{n}\left(\overline{\mathbb{Q}}_{p}\right)$ of highest weight $b_{\tau, 1} \leq \cdots \leq b_{\tau, n}$ relative to the lower triangular Borel. Relative to the upper triangular Borel, $\rho_{\sigma}$ has highest weight $b_{\tau, n} \geq \cdots \geq b_{\tau, 1}$, so that $\rho_{\sigma} \simeq \breve{V}_{a_{\tau}} \otimes \operatorname{det}^{1-n}$ (more precisely, $\bigotimes_{\mathbb{C}, \iota} \overline{\mathbb{Q}}_{p}$ ). Altogether, the algebraic part is

$$
\xi=\bigotimes_{\sigma} \rho_{\sigma} \simeq \bigotimes_{\tau \mid w}\left(\breve{V}_{a_{\tau}} \otimes \operatorname{det}^{1-n}\right)
$$

(the tensor product ranging over $\tau: \mathcal{K} \hookrightarrow \mathbb{C}$ such that $\iota \tau$ induces $w$ ). Here we abuse notation a bit, and use $V_{a_{\tau}}$ to denote the irreducible algebraic representation of $\mathrm{GL}_{n}\left(\overline{\mathbb{Q}}_{p}\right)$ of highest weight $a_{\tau}$. As a representation of $\mathrm{GL}_{n}\left(\mathcal{K}_{w}\right)$, embedded diagonally in $\prod_{\sigma: \mathcal{K}_{w} \hookrightarrow \overline{\mathbb{Q}}_{p}} \mathrm{GL}_{n}\left(\overline{\mathbb{Q}}_{p}\right)$, the algebraic part becomes

$$
\xi=\left(\bigotimes_{\sigma: \mathcal{K}_{w} \hookrightarrow \overline{\mathbb{Q}}_{p}} \breve{V}_{a_{\iota-1} \sigma}\right) \otimes\left(N_{\mathcal{K}_{w} \mid \mathbb{Q}_{p}} \circ \mathrm{det}\right)^{1-n},
$$

which yields the result. 


\section{Completed cohomology}

In this section we will prove the Breuil-Schneider conjecture [2007, Conjecture 4.3], for the potentially semistable representations $\rho=\rho_{\pi, \iota} \mid \Gamma_{\mathcal{K}_{w}}$ above. This will make heavy use of ideas of Emerton [2006b]. The basic idea is to view $\widetilde{\mathrm{BS}}(\rho)$ as a component of the $p$-adic automorphic representation $\tilde{\pi}=\tilde{\pi}_{p} \otimes \pi_{f}^{p}$ attached to $\pi$, which in turn embeds into the completed cohomology $\tilde{H}^{0}$ for $G$.

3.1. The p-adic automorphic representation. We keep our automorphic representation $\pi$ of $U\left(\mathbb{A}_{F}\right)$ of regular weight $a$. Recall that we introduced the group $G=\operatorname{Res}_{F \mid \mathbb{Q}}(U)$. Interchangeably, below we will view $\pi$ as an automorphic representation of $G(\mathbb{A})$. We will follow p. 52 in [Emerton 2006b] in attaching a $p$-adic automorphic representation to $\pi$. (The $\mathbb{G}$ there will be our $G$, and $F$ there will be $\mathbb{Q}$.) This can be done for $W$-allowable $\pi$, where $W$ is an irreducible algebraic representation of $G(\mathbb{C})$, which in this case (where $G$ is compact at infinity) simply means $\left.\pi_{\infty} \simeq W\right|_{G(\mathbb{R})}$. See Definition 3.1.3 in [Emerton 2006b].

To make this more explicit, in terms of the weight $a$, we need to make some identifications. Let us choose a CM-type $\Phi$. For each $\sigma: F \hookrightarrow \mathbb{R}$ we let $\tilde{\sigma}$ denote its lift in $\Phi$. Thus the two extensions to $\mathcal{K}$ are $\left\{\tilde{\sigma}, \widetilde{\sigma}^{c}\right\}$. Via the choice of $\Phi$,

$$
G(\mathbb{C}) \stackrel{\sim}{\longrightarrow} \Phi \mathrm{GL}_{n}(\mathbb{C})^{\operatorname{Hom}(F, \mathbb{R})}, \quad G(\mathbb{R}) \stackrel{\sim}{\longrightarrow} \Phi U(n)^{\operatorname{Hom}(F, \mathbb{R})} .
$$

We immediately infer that $W \simeq \bigotimes_{\sigma \in \operatorname{Hom}(F, \mathbb{R})} \breve{V}_{a_{\widetilde{\sigma}}}$ under these identifications. Via $\iota: \mathbb{C} \stackrel{\sim}{\longrightarrow} \overline{\mathbb{Q}}_{p}$ we identify $W$ with an algebraic representation of $G\left(\overline{\mathbb{Q}}_{p}\right)$. Now

$$
G\left(\overline{\mathbb{Q}}_{p}\right) \stackrel{\sim}{\longrightarrow} \Phi \prod_{v \mid p} \mathrm{GL}_{n}\left(\overline{\mathbb{Q}}_{p}\right)^{\operatorname{Hom}\left(F_{v}, \overline{\mathbb{Q}}_{p}\right)}
$$

allows us to factor our $p$-adic $W$ accordingly, as $W \simeq \bigotimes_{v \mid p} W_{v}$, where we let

$$
W_{v}=\bigotimes_{\substack{\sigma \in \operatorname{Hom}(F, \mathbb{R}) \\ \sigma \mid v}} \breve{V}_{a_{\widetilde{\sigma}}}
$$

In the same vein, $G\left(\mathbb{Q}_{p}\right)=\prod_{v \mid p} U\left(F_{v}\right)$. To go any further, from this point on we assume every $v \mid p$ splits in $\mathcal{K}$, and that $D_{w} \simeq M_{n}\left(\mathcal{K}_{w}\right)$ for each divisor $w \mid v$. Then $U\left(F_{v}\right) \stackrel{\sim}{\longrightarrow} \mathrm{GL}_{n}\left(\mathcal{K}_{w}\right)$, defined up to conjugation. If we assume (as we may) that our CM-type $\Phi$ is ordinary at $\iota$, in the sense of [Katz 1978], then $\Phi$ singles out a place $\tilde{v}$ of $\mathcal{K}$ above each $v \mid p$ of $F$. With this selection of places at hand,

$$
G\left(\mathbb{Q}_{p}\right) \stackrel{\sim}{\longrightarrow} \prod_{v \mid p} \mathrm{GL}_{n}\left(\mathcal{K}_{\tilde{v}}\right) .
$$

Moreover, the inclusion into $G\left(\overline{\mathbb{Q}}_{p}\right)$ corresponds to the diagonal embeddings

$$
\operatorname{GL}_{n}\left(\mathcal{K}_{\tilde{v}}\right)=\mathrm{GL}_{n}\left(F_{v}\right) \hookrightarrow \mathrm{GL}_{n}\left(\overline{\mathbb{Q}}_{p}\right)^{\operatorname{Hom}\left(F_{v}, \overline{\mathbb{Q}}_{p}\right)} .
$$


The following is Definition 3.1.5 in [Emerton 2006b], except that we are working with representations over $\overline{\mathbb{Q}}_{p}$ instead of descending to a finite extension of $\mathbb{Q}_{p}$.

Definition 5. The classical $p$-adic automorphic representation of $G\left(\mathbb{A}_{f}\right)$ over $\overline{\mathbb{Q}}_{p}$ attached to the $W$-allowable automorphic representation $\pi$ of $G(\mathbb{A})$ is

$$
\tilde{\pi}:=\tilde{\pi}_{p} \otimes_{\overline{\mathbb{Q}}_{p}} \pi_{f}^{p}, \quad \tilde{\pi}_{p}:=W \otimes_{\overline{\mathbb{Q}}_{p}} \pi_{p} .
$$

Here $G\left(\mathbb{Q}_{p}\right)$ acts diagonally on $W \otimes_{\bar{Q}_{p}} \pi_{p}$, and $G\left(\mathbb{A}_{f}^{p}\right)$ acts through the second factor $\pi_{f}^{p}$. (Abusing notation, we write $\pi_{p}$ instead of $\pi_{p} \otimes_{\mathbb{C}, l} \overline{\mathbb{Q}}_{p}$ and so on.)

At each $v \mid p$ we introduce $\tilde{\pi}_{v}=W_{v} \otimes_{\overline{\mathbb{Q}}_{p}} \mathrm{BC}_{\tilde{v} \mid v}\left(\pi_{v}\right)$, a locally algebraic representation of $\mathrm{GL}_{n}\left(\mathcal{K}_{\tilde{v}}\right)$, which depends on the choice of an ordinary CM-type $\Phi$. Moreover, $\tilde{\pi}_{p} \simeq \bigotimes_{v \mid p} \tilde{\pi}_{v}$ under the isomorphism $G\left(\mathbb{Q}_{p}\right) \simeq \prod_{v \mid p} \mathrm{GL}_{n}\left(\mathcal{K}_{\tilde{v}}\right)$.

This leads to the main result of this section.

Proposition 6. Suppose every $v \mid p$ of $F$ splits in $\mathcal{K}$, and $D_{w} \simeq M_{n}\left(\mathcal{K}_{w}\right)$ for all $w \mid v$. For each $v \mid p$ of $F$ pick a place $\tilde{v} \mid v$ of $\mathcal{K}$ (this amounts to choosing an $\iota$-ordinary CM-type). Let $\pi$ be an automorphic representation of $U\left(\mathbb{A}_{F}\right)$ of regular weight, and assume $\rho_{\pi, l}$ is (globally) irreducible. Then, for all $v \mid p$ of $F$,

$$
\widetilde{\mathrm{BS}}\left(\left.\rho_{\pi, l}\right|_{\Gamma_{\mathcal{K}_{\tilde{v}}}}\right) \simeq \tilde{\pi}_{v},
$$

which embeds into $\left.\tilde{\pi}\right|_{\mathrm{GL}_{n}\left(\mathcal{K}_{\tilde{v}}\right)}$ (where we restrict via $U\left(F_{v}\right) \stackrel{\sim}{\longrightarrow} \mathrm{GL}_{n}\left(\mathcal{K}_{\tilde{v}}\right)$ ).

Proof. This follows from the preceding discussion, combined with the computation of the Breuil-Schneider representation in Corollary 4 above.

3.2. Algebraic modular forms. We will study the space of modular forms for $G$ of a given weight. To put things in a broader perspective, we will use the cohomological framework of [Emerton 2006b], although we will only work with $H^{0}$, which is explicit and of a combinatorial nature. In our situation, $G(\mathbb{R})$ is compact and connected, so things simplify tremendously, and we only have cohomology in degree zero. Indeed, for every compact open subgroup $K \subset G\left(\mathbb{A}_{f}\right)$, the corresponding arithmetic quotient is a finite set:

$$
Y(K)=G(\mathbb{Q}) \backslash G\left(\mathbb{A}_{f}\right) / K .
$$

An irreducible algebraic representation $W$ of $G(\mathbb{C})$ defines a local system $\mathcal{V}_{W}$ on each $Y(K)$, and $H^{0}\left(Y(K), \mathcal{V}_{\breve{W}}\right)$ is identified with the space of modular forms of level $K$ and weight $W$. That is, all functions $f: G\left(\mathbb{A}_{f}\right) \rightarrow \breve{W}$ which are $K$-invariant on the right and such that $f(\gamma g)=\gamma f(g)$ for all elements $\gamma \in G(\mathbb{Q})$. Then

$$
H^{0}\left(\mathcal{V}_{\breve{W}}\right):=\underset{K}{\lim _{K}} H^{0}\left(Y(K), \mathcal{V}_{\breve{W}}\right) \simeq \bigoplus_{\pi: \pi_{\infty} \simeq W} m_{G}(\pi) \pi_{f}
$$

is a smooth, admissible, semisimple representation of $G\left(\mathbb{A}_{f}\right)$, which we wish to suitably $p$-adically complete. Via our choice of $\iota: \mathbb{C} \stackrel{\sim}{\longrightarrow} \overline{\mathbb{Q}}_{p}$, we will view $W$ as 
a representation of $G\left(\overline{\mathbb{Q}}_{p}\right)$ and so on. Occasionally it will be convenient to work over a field $E \subset \overline{\mathbb{Q}}_{p}$, finite over $\mathbb{Q}_{p}$. It suffices to take $E$ large enough so that it contains the image of every embedding $F \hookrightarrow \overline{\mathbb{Q}}_{p}$. In that case $G$ splits over $E$, and by highest weight theory $W$ may be defined over $E$. Thus, from now on, $H^{0}\left(\mathcal{V}_{\breve{W}}\right)$ is an $E$-vector space with a smooth admissible $G\left(\mathbb{A}_{f}\right)$-action.

Definition 7. For each tame level $K^{p} \subset G\left(\mathbb{A}_{f}^{p}\right)$, following [Emerton 2006b], we introduce

$$
H^{0}\left(K^{p}, \mathcal{O}_{E} / \varpi_{E}^{s}\right):=\underset{K_{p}}{\lim _{n}} H^{0}\left(Y\left(K_{p} K^{p}\right), \mathcal{O}_{E} / \varpi_{E}^{s}\right)
$$

and

$$
\tilde{H}^{0}\left(K^{p}\right):=E \otimes_{\mathcal{O}_{E}} \underset{s}{\lim _{s}} H^{0}\left(K^{p}, \mathcal{O}_{E} / \varpi_{E}^{s}\right)
$$

The latter is an $E$-Banach space with a unitary $G\left(\mathbb{Q}_{p}\right)$-action, commuting with the action of the Hecke algebra $\mathcal{H}\left(K^{p}\right)$ of compactly supported $K^{p}$-biinvariant $E$-valued functions on $G\left(\mathbb{A}_{f}^{p}\right)$. In fact, it becomes a Banach module over the completion $\hat{\mathcal{H}}\left(K^{p}\right)$. Also,

$$
\widetilde{H}^{0}:=\underset{K^{p}}{\lim } \tilde{H}^{0}\left(K^{p}\right),
$$

a locally convex $E$-vector space with an action of $G\left(\mathbb{A}_{f}\right)$.

In our simple setup, they can all be realized very explicitly. For example,

$$
\widetilde{H}^{0}\left(K^{p}\right)=\left\{\text { continuous } Y\left(K^{p}\right) \stackrel{f}{\longrightarrow} E\right\}, \quad Y\left(K^{p}\right)=\underset{K_{p}}{\lim } Y\left(K_{p} K^{p}\right)
$$

with the supremum norm $\|\cdot\|$. The connection to modular forms is via their locally algebraic vectors. We recall their definition:

Definition 8. Let $V$ be a continuous representation of $G\left(\mathbb{Q}_{p}\right)$ over $E$, and let $W$ be an absolutely irreducible algebraic representation of $G\left(\mathbb{Q}_{p}\right)$ over $E$. We assume $E$ is large enough for $\operatorname{End}_{G\left(\mathbb{Q}_{p}\right)}(W)=E$ to hold. The space of locally $W$-algebraic vectors $V_{W \text {-alg }} \subset V$ is the image of the natural "evaluation" map

$$
W \otimes_{E} \operatorname{Hom}_{G\left(\mathbb{Q}_{p}\right)}(W, V) \stackrel{\sim}{\longrightarrow} V_{W \text {-alg }} \subset V .
$$

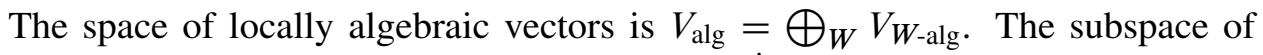

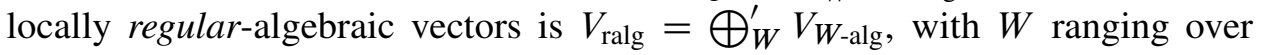
representations of regular weight (in the sense of Section 2.2).

The key ingredient, which relates completed cohomology to modular forms, is the isomorphism: 
Lemma 9. For any absolutely irreducible algebraic representation $W$,

$$
W \otimes_{E} H^{0}\left(\mathcal{V}_{\breve{W}}\right) \stackrel{\sim}{\longrightarrow}\left(\tilde{H}^{0}\right)_{W \text {-alg }}
$$

(When $W$ is only irreducible over $E$, tensor over $\operatorname{End}_{\mathfrak{g}}(W)$, where $\mathfrak{g}=\operatorname{LieG}\left(\mathbb{Q}_{p}\right)$.) Proof. This is Corollary 2.2.25 in [Emerton 2006b] (also spelled out in [Sorensen 2013] for $H^{0}$ ). Let us briefly sketch the main idea. For any tame level $K^{p}$, one shows that

$$
W \otimes_{E} H^{0}\left(K^{p}, \mathcal{V}_{\breve{W}}\right)=\underset{K_{p}}{\underset{\lim }{\longrightarrow}} W \otimes_{E} H^{0}\left(Y\left(K_{p} K^{p}\right), \mathcal{V}_{\breve{W}}\right) \stackrel{\sim}{\longrightarrow} \widetilde{H}^{0}\left(K^{p}\right)_{W \text {-alg }}
$$

This goes as follows: $H^{0}\left(Y\left(K_{p} K^{p}\right), \mathcal{V}_{\breve{W}}\right)$ is a space of classical $p$-adic modular forms, and it is an easy exercise to identify it with $\operatorname{Hom}_{K_{p}}\left(W, \widetilde{H}^{0}\left(K^{p}\right)\right)$. Now,

$$
W \otimes_{E} \operatorname{Hom}_{K_{p}}\left(W, \widetilde{H}^{0}\left(K^{p}\right)\right) \stackrel{\text { eval. }}{\longrightarrow} \widetilde{H}^{0}\left(K^{p}\right)
$$

is injective since $W$ is absolutely irreducible, even when restricted to $K_{p}$ (which is Zariski dense). The image of this evaluation map is the $W$-isotypic subspace of $\tilde{H}^{0}\left(K^{p}\right)$. As $K_{p}$ varies, the maps are compatible, and produces a map out of the direct limit onto $\widetilde{H}^{0}\left(K^{p}\right)_{W \text {-alg, }}$, as desired.

Remark. For higher-degree cohomology $H^{i}$ there is an analogous canonical $G\left(\mathbb{A}_{f}\right)$ -

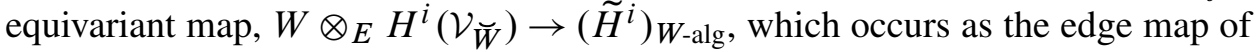
a certain spectral sequence, but the map is not known to be injective for groups other than GL(2) $)_{\mathbb{Q}}$ (and groups $G$ which are compact at infinity modulo center). Injectivity is what makes the whole machinery of [Emerton 2006b] work; see his Theorem 0.7 and Proposition 2.3 .8 on p. 47, for example. In particular, it is available in our case, where $G(\mathbb{R})$ is compact. In general, one would have to localize the spectral sequence at a "cohomologically" non-Eisenstein maximal ideal $\mathfrak{m}$ (which means it does not contribute to mod $p$ cohomology outside the middle degree). This is expected to hold when the Galois representation $\bar{\rho}_{\mathfrak{m}}$ is absolutely irreducible (which is what it means for $\mathfrak{m}$ to be non-Eisenstein), but this is difficult to show. Partial results are now available for $U(2,1)$; see Theorem A in [Emerton and Gee 2013].

From the previous discussion, we get decompositions of completed cohomology: Proposition 10. (1) $\overline{\mathbb{Q}}_{p} \otimes_{E}\left(\tilde{H}^{0}\right)_{W \text {-alg }} \simeq \bigoplus_{\pi: \pi_{\infty} \simeq W} m_{G}(\pi) \tilde{\pi}$.

(2) $\overline{\mathbb{Q}}_{p} \otimes_{E} \tilde{H}^{0}\left(K^{p}\right)_{W \text {-alg }} \simeq \bigoplus_{\pi: \pi_{\infty} \simeq W} m_{G}(\pi)\left(\tilde{\pi}_{p} \otimes_{\overline{\mathbb{Q}}_{p}}\left(\pi_{f}^{p}\right)^{K^{p}}\right)$.

Now, suppose $\mathfrak{h} \subset \mathcal{H}\left(K^{p}\right)$ is a central subalgebra. It then acts on $\left(\pi_{f}^{p}\right)^{K^{p}}$ by a character $\lambda_{\pi}: \mathfrak{h} \rightarrow \overline{\mathbb{Q}}_{p}$. Conversely, say we start out with $\lambda: \mathfrak{h} \rightarrow \overline{\mathbb{Q}}_{p}$. Then,

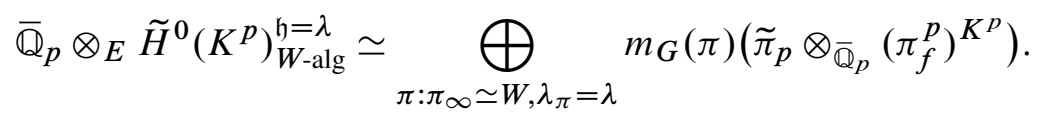


As always, we assume $W$ has regular weight, so we know how to attach Galois representations. If $\mathfrak{h}$ contains the spherical part $\mathcal{H}\left(K^{p}\right)^{\text {sph }}$, all the $\pi$ contributing to the right-hand side have the same Galois representation $\rho_{\lambda}$, by Chebotarev, which we assume is irreducible. By Proposition 6, we may factor the above,

$$
\overline{\mathbb{Q}}_{p} \otimes_{E} \widetilde{H}^{0}\left(K^{p}\right)_{W \text {-alg }}^{\mathfrak{h}=\lambda} \simeq\left(\bigotimes_{v \mid p} \widetilde{\operatorname{BS}}\left(\rho_{\lambda} \mid \Gamma_{\mathcal{K}_{\tilde{v}}}\right)\right) \otimes_{\overline{\mathbb{Q}}_{p}}\left(\bigoplus_{\pi: \pi_{\infty} \simeq W, \lambda_{\pi}=\lambda} m_{G}(\pi) \pi_{f}^{p}\right)^{K^{p}} .
$$

This has the form of a $G\left(\mathbb{Q}_{p}\right) \simeq \prod_{v \mid p} \mathrm{GL}_{n}\left(\mathcal{K}_{\tilde{v}}\right)$-representation tensor an $\mathcal{H}\left(K^{p}\right)$ module. In particular, since $\widetilde{H}^{0}\left(K^{p}\right)$ carries a $G\left(\mathbb{Q}_{p}\right)$-invariant norm, we finally deduce the Breuil-Schneider conjecture for automorphic Galois representations:

Theorem 11. If $\pi$ is an automorphic representation of $U\left(\mathbb{A}_{F}\right)$ of regular weight such that $\rho_{\pi, \iota}$ is irreducible, then $\mathrm{BS}\left(\left.\rho_{\pi, \iota}\right|_{\Gamma_{\mathcal{K}}}\right)$ admits a $\mathrm{GL}_{n}\left(\mathcal{K}_{w}\right)$-invariant norm for all places $w \mid p$ of $\mathcal{K}$.

The discussion leading up to this theorem strongly suggests a better formulation in terms of eigenvarieties. We will employ this machinery in the next section.

\section{Eigenvarieties}

Eigenvarieties are rigid analytic spaces interpolating Hecke eigensystems occurring in spaces of automorphic forms of varying weight. Historically, the first example is the Coleman-Mazur eigencurve for GL(2) $)_{\mathbb{Q}}$, revisited by Buzzard, Emerton, Urban, and others. There are different constructions for any reductive group $G$, which each have their drawbacks and limitations. When $G(\mathbb{R})$ is compact, however, the theory is in good shape, and all constructions are compatible. Below we will combine the approach of [Emerton 2006b] with that of [Chenevier 2009] (for arbitrary totally real $F$ ), extending parts of [Bellaïche and Chenevier 2009] (when $F=\mathbb{Q}$ ).

4.1. The classical points. By our hypotheses, $G_{\mathbb{Q}_{p}} \simeq \prod_{v \mid p} \operatorname{Res}_{\mathcal{K}_{\tilde{v}} \mid \mathbb{Q}_{p}} \operatorname{GL}(n)$ is quasisplit, and we pick the Borel pair $(B, T)$, defined over $\mathbb{Q}_{p}$, corresponding to the product of the upper triangular pairs in each $\mathrm{GL}_{n}\left(\mathcal{K}_{\tilde{v}}\right)$.

As in [Emerton 2006b], let $\widehat{T}$ denote the weight space. That is, the rigid analytic variety (over the coefficient field $E$ introduced in the introduction) which parametrizes the locally analytic characters on $T\left(\mathbb{Q}_{p}\right)$. In other words,

$$
\widehat{T}(A)=\operatorname{Hom}_{l a}\left(T\left(\mathbb{Q}_{p}\right), A^{\times}\right)
$$

for any affinoid $E$-algebra $A$. It comes with a universal map $T\left(\mathbb{Q}_{p}\right) \rightarrow \mathcal{O}(\widehat{T})^{\times}$.

The eigenvariety depends on the choice of tame level $K^{p} \subset G\left(\mathbb{A}_{f}^{p}\right)$, which we will always assume is decomposable as $\prod_{v \nmid p} K_{v}$, where $K_{v}$ is a compact open subgroup of $U\left(F_{v}\right)$ that is hyperspecial for all but finitely many $v$ - say, for 
all $v \notin S\left(K^{p}\right)$. Correspondingly, the Hecke algebra factors as a tensor product,

$$
\mathcal{H}\left(K^{p}\right)=\bigotimes_{v \nmid p} \mathcal{H}\left(K_{v}\right)=\mathcal{H}\left(K^{p}\right)^{\mathrm{ram}} \otimes_{E} \mathcal{H}\left(K^{p}\right)^{\mathrm{sph}} .
$$

Here, $\mathcal{H}\left(K^{p}\right)^{\mathrm{sph}}=\bigotimes_{v \notin S\left(K^{p}\right)} \mathcal{H}\left(K_{v}\right)$ sits as a central subalgebra of $\mathcal{H}\left(K^{p}\right)$; hence it acts by a character on $\pi_{f}^{K^{p}}$ for any automorphic $\pi$ with $K^{p}$-invariants.

We now make precise which points we wish to interpolate by an eigenvariety.

Definition 12. Let $E\left(0, K^{p}\right)_{\mathrm{cl}} \subset\left(\widehat{T} \times \operatorname{Spec} \mathcal{H}\left(K^{p}\right)^{\mathrm{sph}}\right)\left(\overline{\mathbb{Q}}_{p}\right)$ be the subset of pairs $x=(\chi, \lambda)$ for which there exists an irreducible $G\left(\mathbb{A}_{f}\right)$-subquotient $\pi_{f}$ of $\overline{\mathbb{Q}}_{p} \otimes_{E}$ $H^{0}\left(\mathcal{V}_{\breve{W}}\right)$, where $W$ is an irreducible algebraic representation of $G_{E}$, such that:

(a) $\chi=\psi \theta$, where $\psi$ is the highest weight of $W$ (relative to $B$ ), and $\theta$ is a smooth character of $T\left(\mathbb{Q}_{p}\right)$ such that $\pi_{p} \hookrightarrow \operatorname{Ind}_{B\left(\mathbb{Q}_{p}\right)}^{G\left(\mathbb{Q}_{p}\right)}(\theta)$,

(b) $\pi_{f}^{K^{p}} \neq 0$, and $\mathcal{H}\left(K^{p}\right)^{\text {sph }}$ acts on it via $\lambda$.

This is the definition, and notation, used on p. 5 in [Emerton 2006b].

4.2. Eigenvariety conventions. Emerton defines the degree-zero cohomological eigenvariety of $G$, of tame level $K^{p}$, to be the rigid analytic closure of $E\left(0, K^{p}\right)_{\mathrm{cl}}$ in $\widehat{T} \times \operatorname{Spec} \mathcal{H}\left(K^{p}\right)^{\mathrm{sph}}$. By the uniqueness part of Theorem 1.6 in [Chenevier 2009], it coincides with the eigenvariety defined there. We will intertwine the two points of view. Thus, with $E\left(0, K^{p}\right)_{\mathrm{cl}}$ is associated a quadruple $\left(\mathbb{X}, \chi, \lambda, X_{\mathrm{cl}}\right)$, consisting of the following data:

- $\mathbb{X}_{/ E}$ is an equidimensional reduced, rigid, analytic variety,

- $\chi: \mathbb{X} \rightarrow \widehat{T}$ is a finite morphism (Theorem 0.7 (i) on p. 6 in [Emerton 2006b]),

- $\lambda: \mathcal{H}\left(K^{p}\right)^{\mathrm{sph}} \rightarrow \mathcal{O}(\mathbb{X})$ is an $E$-algebra homomorphism,

- $X_{\mathrm{cl}} \subset \mathbb{X}\left(\overline{\mathbb{Q}}_{p}\right)$ is a Zariski-dense subset,

satisfying various properties (listed in Theorem 1.6 in [Chenevier 2009], for example), the most important of which is the following: the canonical evaluation map

$$
\mathbb{X}\left(\overline{\mathbb{Q}}_{p}\right) \longrightarrow\left(\widehat{T} \times \operatorname{Spec} \mathcal{H}\left(K^{p}\right)^{\mathrm{sph}}\right)\left(\overline{\mathbb{Q}}_{p}\right), \quad x \mapsto\left(\chi_{x}, \lambda_{x}\right),
$$

induces a bijection

$$
X_{\mathrm{cl}} \stackrel{\sim}{\longrightarrow} E\left(0, K^{p}\right)_{\mathrm{cl}} .
$$

Moreover, there is a classicality criterion, analogous to Coleman's "noncritical slope implies classical", which we will not use directly (we will use that $X_{\mathrm{cl}}$ is Zariski dense, though). More properties will be recalled below when needed, such as the connection with Emerton's Jacquet functor.

Notation. Following standard usage, by $\mathbb{X}\left(\overline{\mathbb{Q}}_{p}\right)$ we mean the union (or direct limit) of all $\mathbb{X}(L)=\operatorname{Hom}_{E}(\operatorname{Sp}(L), \mathbb{X})$, where $L$ ranges over all the finite extensions of $E$. 
Remark. Loeffler [2011] spells out how Chenevier's construction is related to Emerton's (in the case where $G(\mathbb{R})$ is compact). In addition, he introduces socalled intermediate eigenvarieties, where one replaces $B$ with an arbitrary parabolic subgroup (and drops the assumption that $G$ should be quasisplit at $p$ ). It would be interesting to adapt our arguments to that setting, and thereby make progress towards the Breuil-Schneider conjecture when $\pi_{p}$ does not embed in a principal series (induced from the Borel). This ought to put the results of this paper, and that of [Sorensen 2013], under the same roof. However, at this point we are only producing norms at classical points - where the eigenvariety formalism, strictly speaking, is unnecessary - but the goal is to reach the nonclassical points by somehow $p$-adically varying the norms $\|\cdot\|_{x}$ at classical $x$. We hope to return to these questions elsewhere.

4.3. The Galois pseudocharacter. At each point $x \in \mathbb{X}\left(\overline{\mathbb{Q}}_{p}\right)$ we will assign a continuous semisimple Galois representation $\rho_{x}: \Gamma_{\mathcal{K}} \rightarrow \mathrm{GL}_{n}\left(\overline{\mathbb{Q}}_{p}\right)$, which is unramified outside $\Sigma=\Sigma\left(K^{p}\right)$, the places of $\mathcal{K}$ above $S\left(K^{p}\right)$. This is first done at a dense set of classical points, then by a formal argument one interpolates $\operatorname{tr}\left(\rho_{x}\right)$ by a pseudocharacter. We refer to Chapter 1 of [Bellaïche and Chenevier 2009] for an extensive elegant introduction to pseudorepresentations, a notion going back to Wiles for GL(2), and to Taylor for GL( $n)$.

Definition 13. Let $X_{\text {reg }} \subset X_{\mathrm{cl}}$ be the subset of points $x$ such that $\chi_{x}=\psi_{x} \theta_{x}$, where $\psi_{x}=\bigotimes_{\sigma \in \operatorname{Hom}\left(F, \overline{\mathbb{Q}}_{p}\right)} \psi_{x, \tilde{\sigma}}$ is a regular character of $T$. That is, some $\psi_{x, \tilde{\sigma}}$ is a regular dominant character of $T_{\mathrm{GL}(n)}$ in the usual sense.

This is a Zariski-dense subset of $\mathbb{X}\left(\overline{\mathbb{Q}}_{p}\right)$; see p. 18 in [Chenevier 2009] and the references given there. Now let $x \in X_{\text {reg }}$, and look at the corresponding pair $\left(\chi_{x}, \lambda_{x}\right)$, where $\chi_{x}=\psi_{x} \theta_{x}$. There exists an irreducible $G\left(\mathbb{A}_{f}\right)$-summand $\pi_{f}$ in $\overline{\mathbb{Q}}_{p} \otimes_{E} H^{0}\left(\mathcal{V}_{\breve{W}_{x}}\right)$, where $W_{x}$ has regular highest weight $\psi_{x}$, such that $\mathcal{H}\left(K^{p}\right)^{\text {sph }}$ acts on $\pi_{f}^{K^{p}} \neq 0$ via $\lambda_{x}$, and $\pi_{p} \hookrightarrow \operatorname{Ind}_{B\left(\mathbb{Q}_{p}\right)}^{G\left(\mathbb{Q}_{p}\right)}\left(\theta_{x}\right)$. Thus $\iota^{-1} \pi_{f}$ is the finite part of an automorphic representation of $U\left(\mathbb{A}_{F}\right)$ of regular weight $W_{x}$, unramified outside $S\left(K^{p}\right)$, to which we can associate a continuous semisimple Galois representation

$$
\rho_{x}: \Gamma_{\mathcal{K}} \rightarrow \mathrm{GL}_{n}\left(\overline{\mathbb{Q}}_{p}\right)
$$

with the following properties:

(a) $\rho_{x}^{\vee} \simeq \rho_{x}^{c} \otimes \epsilon_{\text {cyc }}^{n-1}$.

(b) For every finite place $v \nmid p$ of $F$ outside $S\left(K^{p}\right)$, and every $w \mid v$ of $\mathcal{K}$, the local representation $\left.\rho_{x}\right|_{\Gamma_{\mathcal{K}_{w}}}$ is unramified, and satisfies the identity

$$
\operatorname{tr} \rho_{x}\left(\operatorname{Frob}_{w}\right)=\lambda_{x}\left(b_{w \mid v}\left(h_{w}\right)\right) .
$$


(Here, Frob $w$ is a geometric Frobenius and $h_{w}$ is the element of the spherical Hecke algebra for $\mathrm{GL}_{n}\left(\mathcal{K}_{w}\right)$ acting on an unramified $\Pi_{w}$ by $\sum \alpha_{i}$, where the $\alpha_{i}$ are the integral Satake parameters. Finally, the map

$$
b_{w \mid v}: \mathcal{H}\left(\mathrm{GL}_{n}\left(\mathcal{K}_{w}\right), K_{w}\right) \rightarrow \mathcal{H}\left(U\left(F_{v}\right), K_{v}\right)
$$

is the base change homomorphism between the spherical Hecke algebras. See [Mínguez 2011] for a careful useful discussion of this latter map.)

(c) For every finite place $v \mid p$ of $F$, the local representation $\left.\rho_{x}\right|_{\Gamma_{\mathcal{K}_{\tilde{v}}}}$ is potentially semistable. Furthermore:

(i) The semisimplification of the attached Weil-Deligne representation is

$$
\mathrm{WD}\left(\left.\rho_{x}\right|_{\Gamma_{\mathcal{K}}}\right)^{s s} \simeq \bigoplus_{i=1}^{n}\left(\theta_{x, \tilde{v}}^{(i)} \circ \operatorname{Art}_{\mathcal{K}_{\tilde{v}}}^{-1}\right)
$$

(Here $\theta_{x}=\bigotimes_{v \mid p} \theta_{x, \tilde{v}}$, where $\theta_{x, \tilde{v}}$ is a smooth character of the diagonal torus $T_{\mathrm{GL}(n)}\left(\mathcal{K}_{\tilde{v}}\right) \simeq\left(\mathcal{K}_{\tilde{v}}^{*}\right)^{n}$, factored as a product $\left.\theta_{x, \tilde{v}}^{(1)} \otimes \cdots \otimes \theta_{x, \tilde{v}}^{(n)}\right)$

(ii) The Hodge-Tate numbers are, for any embedding $\tau: \mathcal{K}_{\tilde{v}} \hookrightarrow \overline{\mathbb{Q}}_{p}$,

$$
\operatorname{HT}_{\tau}\left(\rho_{x} \mid \Gamma_{\mathcal{K}_{\tilde{v}}}\right)=\left\{a_{\tau, j}+(n-j): j=1, \ldots, n\right\},
$$

where the tuple $\left(a_{\tau, j}\right)$ corresponds to the dominant character $\psi_{x, v, \tau}$ of $T_{\mathrm{GL}(n)}$. (Here we factor $\psi_{x}=\bigotimes_{v \mid p} \otimes_{\tau: \mathcal{K}_{\tilde{v}} \hookrightarrow \overline{\mathbb{Q}}_{p}} \psi_{x, v, \tau}$.)

Observe that there may be many automorphic representations associated to a given point $x \in X_{\mathrm{cl}}$, but they are all isomorphic outside $S\left(K^{p}\right)$ (and of the same weight). In particular, by (b) and Chebotarev, the Galois representation is independent of the choice of $\pi_{f}$, justifying the notation $\rho_{x}$.

Proposition 14. There exists a unique continuous $n$-dimensional pseudocharacter $\mathcal{T}: \Gamma_{\mathcal{K}, \Sigma} \rightarrow \mathcal{O}(\mathbb{X})^{\leq 1}$ such that $\mathcal{T}\left(\operatorname{Frob}_{w}\right)=\lambda\left(b_{w \mid v}\left(h_{w}\right)\right)$ for all places $w \notin \Sigma$.

Proof. We are in the situation of Proposition 7.1.1 in [Chenevier 2004]: $\mathbb{X}$ is reduced, $\mathcal{O}(\mathbb{X})^{\leq 1}$ is a compact subring, and for all $x \in X_{\text {reg }}$, a Zariski-dense subset, we have a representation $\rho_{x}$ of $\Gamma_{\mathcal{K}, \Sigma}$ such that $\operatorname{tr} \rho_{x}\left(\operatorname{Frob}_{w}\right)=\lambda\left(b_{w \mid v}\left(h_{w}\right)\right)(x)$.

Corollary 15. For every $x \in \mathbb{X}\left(\overline{\mathbb{Q}}_{p}\right)$, there is a unique continuous semisimple Galois representation $\rho_{x}: \Gamma_{\mathcal{K}, \Sigma} \rightarrow \mathrm{GL}_{n}\left(\overline{\mathbb{Q}}_{p}\right)$ such that $\operatorname{tr} \rho_{x}\left(\operatorname{Frob}_{w}\right)=\lambda_{x}\left(b_{w \mid v}\left(h_{w}\right)\right)$ for all $w \notin \Sigma$.

Proof. This follows from Theorem 1 of [Taylor 1991].

In particular, this applies to the classical point $x \in X_{\mathrm{cl}}$, not in $X_{\text {reg. }}$. One of the goals of [Chenevier 2009] was to extend properties (a)-(c) above to this setting. This was partially accomplished; see his Theorems 3.3 and 3.5. 


\section{Banach space representations}

With each point $x \in \mathbb{X}(L)$, we have associated an $n$-dimensional continuous pseudocharacter $\mathcal{T}_{x}: \Gamma_{\mathcal{K}} \rightarrow L$, unramified outside $\Sigma\left(K^{p}\right)$. Here we will associate a Banach $\hat{\mathcal{H}}_{L}\left(K^{p}\right)$-module $\mathcal{B}_{x}$, with an admissible unitary $G\left(\mathbb{Q}_{p}\right)$-action, such that the pairs $\left(\mathcal{T}_{x}, \mathcal{B}_{x}\right)$ form the graph of a one-to-one correspondence. We explicitly compute the locally (regular) algebraic vectors in $\mathcal{B}_{x}$ for $x \in X_{\text {reg }}$ such that $\mathcal{T}_{x}$ is absolutely irreducible, in terms of the Breuil-Schneider representation attached to $\mathcal{T}_{x}$, or rather, its corresponding Galois representation $\rho_{x}$. As a result, we prove the Breuil-Schneider conjecture for such $\rho_{x}$.

A global p-adic Langlands correspondence. With the eigenvariety language set up, we can reformulate our findings at the end of Section 2. We let $X_{\text {irr }} \subset \mathbb{X}\left(\overline{\mathbb{Q}}_{p}\right)$ be the points $x$ for which $\rho_{x}$ is irreducible.

Theorem 16. Let $x \in X_{\mathrm{reg}} \cap X_{\mathrm{irr}}$, corresponding to $\left(\psi_{x} \theta_{x}, \lambda_{x}\right)$. Let $W_{x}$ be the irreducible algebraic representation of $G_{E}$ of highest weight $\psi_{x}$. Then,

$$
\overline{\mathbb{Q}}_{p} \otimes_{E} \widetilde{H}^{0}\left(K^{p}\right)_{W_{x} \text {-alg }}^{\mathfrak{h}=\lambda_{x}} \simeq\left(\bigoplus_{v \mid p} \widetilde{\operatorname{BS}}\left(\left.\rho_{x}\right|_{\Gamma_{\mathcal{K}_{\tilde{v}}}}\right)\right) \otimes_{\overline{\mathbb{Q}}_{p}}\left(\underset{\substack{\pi: \pi_{\infty} \simeq W_{x}, \lambda_{\pi}=\lambda_{x}}}{\bigoplus} m_{G}(\pi)\left(\pi_{f}^{p}\right)^{K^{p}}\right),
$$

where we write $\mathfrak{h}=\mathcal{H}\left(K^{p}\right)^{\mathrm{sph}}$ for simplicity.

This formula suggests the following definition.

Definition 17. At each point $x \in \mathbb{X}\left(\overline{\mathbb{Q}}_{p}\right)$, we introduce the eigenspace

$$
\mathcal{B}_{x}:=\left(\overline{\mathbb{Q}}_{p} \otimes_{E} \tilde{H}^{0}\left(K^{p}\right)\right)^{\mathfrak{h}=\lambda_{x}} .
$$

This is a Banach $\hat{\mathcal{H}}\left(K^{p}\right)$-module with a (commuting) unitary $G\left(\mathbb{Q}_{p}\right)$-action.

We remind ourselves that $\mathcal{B}_{x}$ is nothing but the space of continuous $\lambda_{x}$-eigenforms $f: Y\left(K^{p}\right) \rightarrow \overline{\mathbb{Q}}_{p}$. This sets up a one-to-one correspondence $\rho_{x} \leftrightarrow \mathcal{B}_{x}$. That is,

$$
\rho_{x}=\rho_{x^{\prime}} \Longleftrightarrow \lambda_{x}=\lambda_{x^{\prime}} \Longleftrightarrow \mathcal{B}_{x}=\mathcal{B}_{x^{\prime}}
$$

for any two $x, x^{\prime} \in \mathbb{X}\left(\overline{\mathbb{Q}}_{p}\right)$. Let us say that a Galois representation $\rho$ comes from $\mathbb{X}$ if $\rho \simeq \rho_{x}$ for some $x \in \mathbb{X}\left(\overline{\mathbb{Q}}_{p}\right)$, and similarly for Banach modules $\mathcal{B} \simeq \mathcal{B}_{x}$.

This leads to the main result of this section, which in some sense is the genesis of what follows.

Theorem 18. The eigenvariety $\mathbb{X}$ mediates a one-to-one correspondence between:

- The set of continuous semisimple Galois representations $\rho: \Gamma_{\mathcal{K}} \rightarrow \mathrm{GL}_{n}\left(\overline{\mathbb{Q}}_{p}\right)$ coming from $\mathbb{X}$. (In particular, $\rho$ is unramified outside $\Sigma\left(K^{p}\right)$.)

- The set of Banach $\hat{\mathcal{H}}\left(K^{p}\right)$-modules $\mathcal{B}$, with unitary $G\left(\mathbb{Q}_{p}\right)$-action, from $\mathbb{X}$.

We write $\rho \leftrightarrow \mathcal{B}$ when there is a point $x \in \mathbb{X}\left(\overline{\mathbb{Q}}_{p}\right)$ such that $\rho \simeq \rho_{x}$ and $\mathcal{B} \simeq \mathcal{B}_{x}$. 


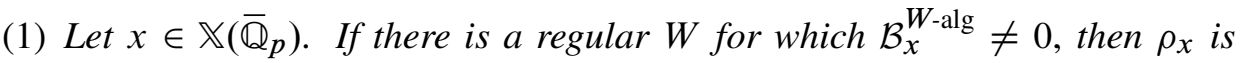
potentially semistable at all places $w \mid p$ of $\mathcal{K}$.

(2) Let $x \in X_{\mathrm{cl}}$. Then $\mathcal{B}_{x}^{W_{x} \text {-alg }} \neq 0$, and $\mathcal{B}_{x}^{W \text {-alg }}=0$ for all regular $W \neq W_{x}$.

(3) For $x \in X_{\text {reg }} \cap X_{\text {irr }}$, the locally regular-algebraic vectors of $\mathcal{B}_{x}$ are

$$
\mathcal{B}_{x}^{\mathrm{ralg}}=\mathcal{B}_{x}^{W_{x} \text {-alg }} \simeq\left(\bigotimes_{v \mid p} \widetilde{\mathrm{BS}}\left(\rho_{x} \mid \Gamma_{\mathcal{K}_{\tilde{v}}}\right)\right) \otimes_{\overline{\mathbb{Q}}_{p}}\left(\bigoplus_{\substack{\pi: \pi_{\infty} \simeq W_{x} \\ \lambda_{\pi}=\lambda_{x}}} m_{G}(\pi)\left(\pi_{f}^{p}\right)^{K^{p}}\right)
$$

Remark. This is a strengthening of Theorem 11, which in the notation of the above Theorem 18 merely says that $\widetilde{\mathrm{BS}}\left(\rho_{x} \mid \Gamma_{\mathcal{K}_{\tilde{v}}}\right)$ admits an invariant norm. We stress that $x$ is a classical point here (so that $\rho_{x}$ is irreducible, of regular weight). Thus $\rho_{x} \simeq \rho_{\pi, \iota}$ for an automorphic $\pi$ as in Theorem 11. Part (3) of Theorem 18 is stronger, in that it makes precise how $\widetilde{\mathrm{BS}}\left(\left.\rho_{x}\right|_{\Gamma_{\mathcal{K}_{\tilde{v}}}}\right)$ factors into the Banach representation $\mathcal{B}_{x}$ of $p$-adic modular forms. Another key point is that Theorem 18 emphasizes the correspondence $\rho_{x} \leftrightarrow \mathcal{B}_{x}$, which is defined for all points $x$ on the eigenvariety $\mathbb{X}$ (classical or not).

Proof. First, (1) follows from Proposition 10, which shows there is an automorphic $\pi$, with $\pi_{\infty} \simeq W$, such that $\mathfrak{h}$ acts on $\pi_{f}^{K^{p}}$ by $\lambda_{x}$. Since $W$ is regular, we know how to associate a Galois representation $\rho_{\pi, \iota}$ with the usual local properties, which must be $\rho_{x}$ by Tchebotarev.

For (2), we follow the same line of argument. Since $x \in X_{\mathrm{cl}}$, there is an automorphic $\pi$ contributing to $\mathcal{B}_{x}^{W_{x} \text {-alg }}$. Moreover, if $\mathcal{B}_{x}^{W \text {-alg }} \neq 0$, there is an automorphic $\pi$, of regular weight $W$, for which $\rho_{\pi, \iota} \simeq \rho_{x}$. From $\rho_{\pi, \iota}$ we can recover $W$ through its Hodge-Tate numbers, and similarly for $\rho_{x}$, even if $x$ is not in $X_{\text {reg }}$ (this is shown in Section 3.15 of [Chenevier 2009], based on results of Sen, Berger and Colmez). Therefore, $W=W_{x}$.

Remark. As remarked earlier, we are optimistic that one can remove the regularity hypotheses in the theorem. Indeed it seems possible to attach Galois representations to automorphic $\pi$ of $U\left(\mathbb{A}_{F}\right)$ of irregular weight. When $\pi_{p}$ is of finite slope (that is, embeds in a principal series), this can be done by means of eigenvarieties, as in [Chenevier 2009]. In general, it seems likely that one can push the ideas from the proof of Theorem 1. By [White 2012], there is always a base change $\boxplus_{i=1}^{t} \Pi_{i}$, where the $\Pi_{i}$ are discrete automorphic representations of $\operatorname{GL}_{n_{i}}\left(\mathbb{A}_{\mathcal{K}}\right)$, which in turn (by the Moeglin-Waldspurger classification) are isobaric sums of cohomological, essentially conjugate, self-dual cusp forms, with which one can associate Galois representations. Local-global compatibility at $p$ follows from [Caraiani 2012]. 


\section{Compatibility with classical local Langlands}

In this section we deduce from our previous results that $\widetilde{\mathrm{BS}}\left(\rho_{x}\right)$ admits an invariant norm such that the completion $\widetilde{\mathrm{BS}}\left(\rho_{x}\right)^{\wedge}$ satisfies local-global compatibility (see Corollary 19 below for the precise statement). However, we cannot show that this completion $\widetilde{\mathrm{BS}}\left(\rho_{x}\right)^{\wedge}$ only depends on the restrictions of $\rho_{x}$ to places above $p-$ which seems to be an extremely difficult problem at the heart of the $p$-adic Langlands program. In Section 6.2 we will restrict ourselves to the unramified case, and prove a "weak" version of local-global compatibility (somewhat similar to part (1) of Theorem 1.2.1 in [Emerton 2011]) — it is "weak" since we only get a nonzero map (with a huge kernel) instead of an embedding. We refer the reader to part (b) of Theorem 21: the $p$-adic local Langlands correspondence, still mysterious in higher rank, is replaced by the coarse version in [Schneider and Teitelbaum 2006], which associates a huge Banach representation $B_{\xi, \zeta}$ with a pair $(\xi, \zeta)$ satisfying the Emerton condition (here $\xi$ is an irreducible algebraic representation, and $\zeta$ is a suitable Weyl-orbit in the dual torus). The philosophy propounded in [Schneider and Teitelbaum 2006; Breuil and Schneider 2007] is that the (almost) quotients of $B_{\xi, \zeta}$ should somehow correspond to the crystalline representations of type $(\xi, \zeta)$. This is well understood for $\mathrm{GL}_{2}\left(\mathbb{Q}_{p}\right)$, where the admissible filtration is usually unique (see Theorem 2.3.2, p. 8, of Berger's [2011] survey), and $B_{\xi, \zeta}$ essentially is the local $p$-adic Langlands correspondence in the (irreducible) crystalline case. We provide evidence supporting this philosophy of Breuil, Schneider, and Teitelbaum for $n>2$.

\subsection{Completions of the space of algebraic vectors.}

Split ramification and the automorphic representation $\pi_{x}$. Throughout, we will make the assumption that we have split ramification. That is, $S\left(K^{p}\right) \subset \operatorname{Spl}_{\mathcal{K} \mid F}$. This has the effect that the local base change $\mathrm{BC}_{w \mid v}$ is defined at all places $v$. We fix a point $x \in X_{\text {reg }} \cap X_{\text {irr }}$, as above. Under our ramification hypothesis, there is a unique automorphic representation $\pi$ of $U\left(\mathbb{A}_{F}\right)$ contributing to the (regular) algebraic vectors $\mathcal{B}_{x}^{\text {ralg }}$ in Theorem 18(3). Indeed, any such $\pi$ has an irreducible Galois representation $\rho_{\pi, \iota} \simeq \rho_{x}$, and therefore $\mathrm{BC}_{\mathcal{K} \mid F}(\pi)$ must be cuspidal, and it is uniquely determined at the infinite places, and away from $\Sigma\left(K^{p}\right)$. By strong multiplicity one for $\mathrm{GL}_{n}$, the base change is unique. Locally, $\mathrm{BC}_{w \mid v}$ is injective (see Corollary 4.2 in [Mínguez 2011]), and therefore $\pi$ is uniquely determined. We denote it $\pi_{x}=\bigotimes \pi_{x, v}$. Its local components $\pi_{x, v}$ are given by

$$
\mathrm{WD}\left(\left.\rho_{x}\right|_{\Gamma_{\mathcal{K}_{w}}}\right)^{\mathrm{F}-\mathrm{ss}} \simeq \operatorname{rec}\left(\mathrm{BC}_{w \mid v}\left(\pi_{x, v}\right) \otimes|\operatorname{det}|_{w}^{(1-n) / 2}\right) .
$$

We think of $\left\{\pi_{x}\right\}$ as a family of automorphic representations interpolated by $\mathbb{X}$. In general (without split ramification) the $\pi_{x}$ will be $L$-packets, not singletons. 
With this notation, part (3) of Theorem 18 becomes: for all $x \in X_{\mathrm{reg}} \cap X_{\mathrm{irr}}$,

$$
\mathcal{B}_{x}^{\mathrm{ralg}} \simeq \widetilde{\mathrm{BS}}\left(\rho_{x}\right) \otimes\left(\bigotimes_{v \nmid p} \pi_{x, v}^{K_{v}}\right)^{m\left(\pi_{x}\right)} .
$$

Most likely, $m\left(\pi_{x}\right)=1$, and this may already be in the literature. However, we have not been able to find a suitable reference. Now, since $\bigotimes_{v \nmid p} \pi_{x, v}^{K_{v}}$ is a simple $\mathcal{H}\left(K^{p}\right)$-module, we may think of $\widetilde{\mathrm{BS}}\left(\rho_{x}\right)^{m\left(\pi_{x}\right)}$ as its multiplicity space in $\mathcal{B}_{x}^{\mathrm{ralg}}$,

$$
\widetilde{\mathrm{BS}}\left(\rho_{x}\right)^{m\left(\pi_{x}\right)} \stackrel{\sim}{\longrightarrow} \operatorname{Hom}_{\mathcal{H}\left(K^{p}\right)}\left(\bigotimes_{v \nmid p} \pi_{x, v}^{K_{v}, \mathcal{B}_{x}^{\text {ralg }}}\right),
$$

as representations of $G\left(\mathbb{Q}_{p}\right)$. We will view the right-hand side as sitting inside a Banach space of continuous transformations. For that purpose, we first look at each local component $\pi_{x, v}$, where $v \nmid p$. When $v$ splits, it can be identified with a $p$ integral irreducible representation of $\mathrm{GL}_{n}\left(F_{v}\right)$. By Theorem 1 in [Vignéras 2004], it has a unique commensurability class of stable lattices. Correspondingly, $\pi_{x, v}$ has a unique equivalence class of $\mathrm{GL}_{n}\left(F_{v}\right)$-invariant norms $\|\cdot\|_{v}$. (By Theorem 1 in [Vignéras 2010], the completion $\hat{\pi}_{x, v}$ is a topologically irreducible unitary Banach space representation of $\mathrm{GL}_{n}\left(F_{v}\right)$.) When $\pi_{x, v}$ is unramified, its Satake parameters are $p$-units, and one easily finds a stable lattice in a suitable unramified principal series, again resulting in a $U\left(F_{v}\right)$-invariant (supremum) norm $\|\cdot\|_{v}$, which we may normalize so that a given spherical vector has norm one. The tensor product norm (see Proposition 17.4 in [Schneider 2002]) on $\bigotimes_{v \nmid p} \pi_{x, v}$ is then invariant under $G\left(\mathbb{A}_{f}^{p}\right)$. By restriction, the finite-dimensional space $\bigotimes_{v \nmid p} \pi_{x, v}^{K_{v}}$ inherits a norm, and becomes a Banach-module for $\widehat{\mathcal{H}}\left(K^{p}\right)$. With this extra structure at hand,

$$
\operatorname{Hom}_{\mathcal{H}\left(K^{p}\right)}\left(\bigotimes_{v \nmid p} \pi_{x, v}^{K_{v}, \mathcal{B}_{x}^{\text {ralg }}}\right) \hookrightarrow \mathcal{L}_{\hat{\mathcal{H}}\left(K^{p}\right)}\left(\bigotimes_{v \nmid p} \pi_{x, v}^{K_{v}, \mathcal{B}_{x}}\right) .
$$

(Here $\mathcal{L}$ denotes the space of continuous linear transformations equipped with the usual transformation norm; see Corollary 3.2 in [Schneider 2002].) We have to check that any $\mathcal{H}\left(K^{p}\right)$-equivariant map $\bigotimes_{v \nmid p} \pi_{x, v}^{K_{v}} \stackrel{\phi}{\longrightarrow} \mathcal{B}_{x}$ is automatically continuous. If $\phi \neq 0$, it must be injective (by simplicity), and thus $\|\phi(\cdot)\|_{\mathcal{B}_{x}}$ defines a norm on $\bigotimes_{v \nmid p} \pi_{x, v}^{K_{v}}$. However, all norms on a finite-dimensional space are equivalent (Proposition 4.13 in [Schneider 2002]), so that $\|\phi(u)\|_{\mathcal{B}_{x}} \leq C\|u\|$ for some constant $C>0$ and all $u$. Altogether, this embeds $\widetilde{\mathrm{BS}}\left(\rho_{x}\right)$ into a Banach space (Proposition 3.3 in [Schneider 2002]):

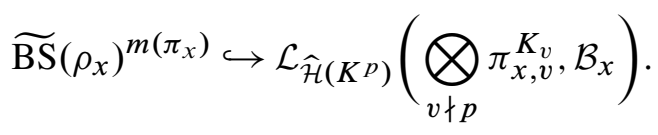

If we restrict the transformation norm to $\widetilde{\mathrm{BS}}\left(\rho_{x}\right)^{m\left(\pi_{x}\right)}$, we arrive at: 
Corollary 19. Let $x \in X_{\text {reg }} \cap X_{\text {irr }}$ be a point such that $m\left(\pi_{x}\right)=1$. Then there is a $G\left(\mathbb{Q}_{p}\right)$-invariant norm $\|\cdot\|$ on $\widetilde{\mathrm{BS}}\left(\rho_{x}\right)$ such that the corresponding completion $\widetilde{\mathrm{BS}}\left(\rho_{x}\right)^{\wedge}$ satisfies the following: There is a topological isomorphism

$$
\widetilde{\mathrm{BS}}\left(\rho_{x}\right)^{\wedge} \otimes\left(\bigotimes_{v \nmid p} \pi_{x, v}^{K_{v}}\right) \stackrel{\sim}{\longrightarrow} \overline{\mathcal{B}_{x}^{\mathrm{ralg}}},
$$

where $\overline{\mathcal{B}_{x}^{\text {ralg }}}$ is the closure of the regular-algebraic vectors $\mathcal{B}_{x}^{\text {ralg }}$ in $\mathcal{B}_{x}$. Moreover:

- $\widetilde{\mathrm{BS}}\left(\rho_{x}\right)^{\wedge}$ is an admissible unitary Banach space representation of $G\left(\mathbb{Q}_{p}\right)$.

- Its regular-algebraic vectors $\widetilde{\mathrm{BS}}\left(\rho_{x}\right)$ form a dense subspace.

Proof. We obtain $\|\cdot\|$ by restricting the transformation norm to $\widetilde{\mathrm{BS}}\left(\rho_{x}\right)$. Thus (1) becomes an isometry, and extends uniquely to an isometry

$$
\widetilde{\mathrm{BS}}\left(\rho_{x}\right)^{\wedge} \hookrightarrow \mathcal{L}_{\hat{\mathcal{H}}\left(K^{p}\right)}\left(\bigotimes_{v \nmid p} \pi_{x, v}^{K_{v}}, \mathcal{B}_{x}\right) .
$$

To ease the notation, let us write $M=\bigotimes_{v \nmid p} \pi_{x, v}^{K_{v}}$ throughout this proof; $M$ is a finite-dimensional simple $\mathcal{H}\left(K^{p}\right)$-module. We tensor the isometry by this $M$ :

$$
j: \widetilde{\mathrm{BS}}\left(\rho_{x}\right)^{\wedge} \otimes M \hookrightarrow \mathcal{L}_{\widehat{\mathcal{H}}\left(K^{p}\right)}\left(M, \mathcal{B}_{x}\right) \otimes M \stackrel{\sim}{\longrightarrow} \mathcal{B}_{x}[M] .
$$

Here $\mathcal{B}_{x}[M]$ denotes the closure of the sum of all closed $\mathcal{H}\left(K^{p}\right)$-submodules of $\mathcal{B}_{x}$ isomorphic to $M$ (a topological direct sum of a subcollection, by Zorn). Note that $\operatorname{End}_{\mathcal{H}\left(K^{p}\right)}(M)=\overline{\mathbb{Q}}_{p}$. Note also that the tensor products (equipped with their tensor product norms, as on p. 110 in [Schneider 2002]), are already complete, as $M$ is finite-dimensional. The above isomorphism with $\mathcal{B}_{x}[M]$ is a topological isomorphism by the open mapping theorem (Proposition 8.6 in [Schneider 2002]), but not necessarily isometric. Consequently, $\operatorname{im}(j) \subset \mathcal{B}_{x}$ is a closed subspace, containing $\mathcal{B}_{x}^{\text {ralg }}$ by Theorem 18. In fact, $\operatorname{im}(j)$ is the closure of $\mathcal{B}_{x}^{\text {ralg }}$ in $\mathcal{B}_{x}$, since $\widetilde{\mathrm{BS}}\left(\rho_{x}\right)$ is dense in the completion $\widetilde{\mathrm{BS}}\left(\rho_{x}\right)^{\wedge}$. Again invoke the open mapping

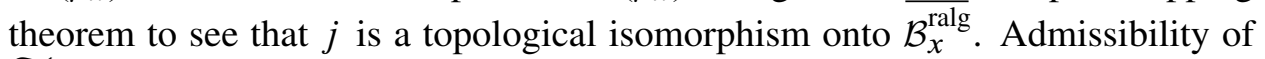
$\widetilde{\mathrm{BS}}\left(\rho_{x}\right)^{\wedge}$ follows from admissibility of $\mathcal{B}_{x}$.

Remark. Equivalently, there is a $G\left(\mathbb{Q}_{p}\right)$-equivariant topological isomorphism

$$
\widetilde{\operatorname{BS}}\left(\rho_{x}\right)^{\wedge} \stackrel{\sim}{\longrightarrow} \mathcal{L}_{\hat{\mathcal{H}}\left(K^{p}\right)}\left(\bigotimes_{v \nmid p} \pi_{x, v}^{K_{v}}, \overline{\mathcal{B}_{x}^{\mathrm{ralg}}}\right) .
$$

We like to think of this Banach space representation $\widetilde{\mathrm{BS}}\left(\rho_{x}\right)^{\wedge}$ as a rough candidate for a $p$-adic local Langlands correspondence, at least when the various restrictions $\left.\rho_{x}\right|_{\Gamma_{\mathcal{K}}}$ are irreducible. Of course, to really justify this point of view, one would need to show that the completion $\widetilde{\mathrm{BS}}\left(\rho_{x}\right)^{\wedge}$ only depends on the restrictions $\left.\rho_{x}\right|_{\Gamma_{\mathcal{K}}}$ 
at $p$, and that it factors as a tensor product $\hat{\bigotimes}_{v \mid p}$ of appropriate completions $\widetilde{\mathrm{BS}}\left(\left.\rho_{x}\right|_{\Gamma_{\mathcal{K}_{\tilde{v}}}}\right)^{\wedge}$. Both appear to be very difficult questions.

6.2. Universal modules and the crystalline case. We now specialize to the crystalline case, where we can relate $\widetilde{\mathrm{BS}}\left(\rho_{x}\right)^{\wedge}$ to the Schneider-Teitelbaum universal modules $B_{\xi, \zeta}$, which are given by a purely local construction at $p$. They are expected to be quite large. However, for $n>2$ it is not even known that $B_{\xi, \xi} \neq 0$ (Conjecture 6.1, p. 24 in [Breuil and Schneider 2007]). For $n=2$ this is a deep result of Berger and Breuil. We will prove nonvanishing when $(\xi, \zeta)$ "comes from an eigenvariety". This will be a by-product of a stronger result.

Definition 20. A classical point $x \in X_{\mathrm{cl}}$ is called old if $\rho_{x}$ is crystalline at all places above $p$. That is, $\operatorname{Hom}_{G\left(\mathbb{Z}_{p}\right)}\left(W_{x}, \mathcal{B}_{x}\right) \neq 0$. Equivalently, $\pi_{x, v}$ is unramified for all $v \mid p$. We denote the set of old points by $X_{\text {old }}$.

Thus, from now on, we fix a point $x \in X_{\text {reg }} \cap X_{\text {irr }} \cap X_{\text {old }}$. By Proposition 6,

$$
\widetilde{\mathrm{BS}}\left(\rho_{x}\right)=W_{x} \otimes \pi_{x, p} \stackrel{\sim}{\longrightarrow} W_{x} \otimes \operatorname{Ind}_{B}^{G}\left(\theta_{x}\right),
$$

where $\theta_{x}$ is unramified smooth. (Indeed, for any point $x, \pi_{x, p}$ embeds into the (unnormalized) principal series $\operatorname{Ind}_{B}^{G}\left(\theta_{x}\right)$. Since $x$ is old, $\pi_{x, p}$ is unramified, and hence so is $\theta_{x}$. Furthermore, as $x \in X_{\text {irr }}$, the base change $\mathrm{BC}_{\mathcal{K} \mid F}\left(\pi_{x}\right)$ is cuspidal, and therefore generic. In particular, $\pi_{x, p}$ must be generic. As is well known, this implies that $\pi_{x, p}$ must be the full unramified principal series.)

As in [Schneider and Teitelbaum 2006], we express $\pi_{x, p} \simeq \operatorname{Ind}_{B}^{G}\left(\theta_{x}\right)$ in terms of the universal module. This goes back to Borel and Matsumoto, and is defined as follows. For any algebra character $\zeta: \mathcal{H}(G, K) \rightarrow \overline{\mathbb{Q}}_{p}$ (where $K=G\left(\mathbb{Z}_{p}\right)$ is hyperspecial when $p$ is assumed to be unramified in $F$ ) we introduce the smooth representation

$$
\mathcal{M}_{\zeta}=\mathrm{c}-\operatorname{Ind}_{K}^{G}(1) \otimes_{\mathcal{H}(G, K), \zeta} \overline{\mathbb{Q}}_{p}=\mathcal{C}_{c}\left(K \backslash G, \overline{\mathbb{Q}}_{p}\right) \otimes_{\mathcal{H}(G, K), \zeta} \overline{\mathbb{Q}}_{p} .
$$

The pair $\left(\mathcal{M}_{\zeta}, 1_{K}\right)$ is a universal initial object in the category of pairs $(V, v)$, where $V$ is an unramified smooth representation of $G\left(\mathbb{Q}_{p}\right)$, and $v \in V^{K}$ is a nonzero vector on which $\mathcal{H}(G, K)$ acts via $\zeta$. That is, there is a unique $G\left(\mathbb{Q}_{p}\right)$-map $\mathcal{M}_{\zeta} \rightarrow V$ which maps $1_{K} \mapsto v$. The image of this map is the span of the orbit $G v$ (since $\mathcal{M}_{\zeta}$ is generated by $1_{K}$ ). In what follows we will take $\zeta_{x}=\hat{\theta}_{x}$, the eigensystem of $\operatorname{Ind}_{B}^{G}\left(\theta_{x}\right)^{K}$. The choice of a spherical vector yields

$$
\mathcal{M}_{\zeta_{x}} \rightarrow \operatorname{Ind}_{B}^{G}\left(\theta_{x}\right), \quad \zeta_{x}=\hat{\theta}_{x} .
$$

It is a general fact that the two representations have the same semisimplification (see the Ph.D. thesis of X. Lazarus [2000] for a thorough discussion in greater 
generality). Under our assumptions, $\operatorname{Ind}_{B}^{G}\left(\theta_{x}\right)$ is irreducible, and therefore the above must be an isomorphism. Consequently, we may identify

$$
\widetilde{\mathrm{BS}}\left(\rho_{x}\right) \simeq W_{x} \otimes \mathcal{M}_{\zeta_{x}} \simeq \mathrm{c}-\operatorname{Ind}_{K}^{G}\left(\xi_{x}\right) \otimes_{\mathcal{H}_{\xi x}(G, K), \zeta_{x}} \overline{\mathbb{Q}}_{p}=: H_{\xi_{x}, \zeta_{x}} .
$$

Here we have changed notation $\xi_{x}:=W_{x}$ to aid comparison with [Schneider and Teitelbaum 2006]. The algebra $\mathcal{H}_{\xi_{x}}(G, K)$ consists, by definition, of the $G$-endomorphisms of c-Ind $G_{K}\left(\xi_{x}\right)$; or, more concretely, of compactly supported $K$-biequivariant functions $G \rightarrow \operatorname{End}\left(\xi_{x}\right)$ with convolution. However, since $\xi$ is an irreducible representation of $G$ (viewed as a representation of $K$ ), as on p. 639 in [Schneider and Teitelbaum 2006] one can identify the algebras

$$
\mathcal{H}(G, K) \stackrel{\sim}{\longrightarrow} \mathcal{H}_{\xi_{x}}(G, K), \quad h \mapsto\left(g \mapsto h(g) \xi_{x}(g)\right) .
$$

In the definition of $H_{\xi_{x}}, \zeta_{x}$ we view $\zeta_{x}$ as a character of $\mathcal{H}_{\xi_{x}}(G, K)$ via this isomorphism, as at the bottom of p. 670 in [Schneider and Teitelbaum 2006], where $H_{\xi, \zeta}$ is defined.

The representation $H_{\xi_{x}}, \zeta_{x}$ has a natural locally convex topology, being a quotient of c-Ind $G_{K}\left(\xi_{x}\right)$, which has a supremum norm: Pick any norm $\|\cdot\|_{\xi_{x}}$ on $\xi_{x}$ which is invariant under (the compact group) $K$. They are all equivalent since $\xi_{x}$ is finite-dimensional (Proposition 4.13 in [Schneider 2002]). For $f \in{\mathrm{c}-\operatorname{Ind}_{K}}^{G}\left(\xi_{x}\right)$, we let

$$
\|f\|_{\xi_{x}, \infty}=\sup _{g \in G\left(\mathbb{Q}_{p}\right)}\|f(g)\|_{\xi_{x}}<\infty
$$

This defines an norm $\|\cdot\|_{\xi_{x}, \infty}$ on the compact induction, which is obviously invariant under $G\left(\mathbb{Q}_{p}\right)$, and it induces a quotient seminorm on the representation

$$
H_{\xi_{x}, \zeta_{x}}=\left(\mathrm{c}-\operatorname{Ind}_{K}^{G}\left(\xi_{x}\right)\right) /\left(\operatorname{ker} \zeta_{x}\right)\left(\mathrm{c}-\operatorname{Ind}_{K}^{G}\left(\xi_{x}\right)\right) .
$$

We will show below that in fact this is a norm, but this is far from clear a priori!

Following [Schneider and Teitelbaum 2006], on p. 671 where they define $B_{\xi, \zeta}$, we introduce the space

$$
B_{\xi_{x}, \zeta_{x}}:=\widehat{H}_{\xi_{x}, \zeta_{x}}=\left(H_{\xi_{x}, \zeta_{x}} / \overline{\{0\}}\right)^{\wedge},
$$

the Hausdorff completion of $H_{\xi_{x}}, \zeta_{x}$. (We refer to Proposition 7.5 in [Schneider 2002] for a general discussion of Hausdorff completions.) We have defined a Banach space $B_{\xi_{x}}, \zeta_{x}$ with a unitary $G\left(\mathbb{Q}_{p}\right)$-action. However, it is not clear at all that it is nonzero. This is in fact a fundamental problem! Conjecture 6.1 on p. 24 in [Breuil and Schneider 2007] says that $B_{\xi, \zeta} \neq 0$ whenever the Emerton condition is satisfied (the converse is known). This follows from our methods when the pair $(\xi, \zeta)$ comes from an eigenvariety, that is, when it is of the form $\left(\xi_{x}, \zeta_{x}\right)$ for an old irreducible point $x$. What we prove is a strengthening: 
Theorem 21. Let $x \in X_{\mathrm{reg}} \cap X_{\mathrm{irr}} \cap X_{\text {old }}$ be a classical point such that $m\left(\pi_{x}\right)=1$. Then $H_{\xi_{x}}, \zeta_{x}$ is Hausdorff and $B_{\xi_{x}}, \zeta_{x} \neq 0$ is its universal completion. Furthermore:

(a) There is a continuous map, with dense image, $B_{\xi_{x}, \xi_{x}} \rightarrow \widetilde{\mathrm{BS}}\left(\rho_{x}\right)^{\wedge}$ (into the com-

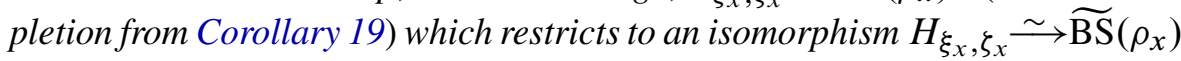
onto the regular-algebraic vectors.

(b) There is a nonzero $G\left(\mathbb{Q}_{p}\right) \times \widehat{\mathcal{H}}\left(K^{p}\right)$-equivariant continuous map

$$
B_{\xi_{x}, \zeta_{x}} \otimes\left(\bigotimes_{v \nmid p} \pi_{x, v}^{K_{v}}\right) \rightarrow \overline{\mathcal{B}_{x}^{\text {ralg }}}
$$

with dense image.

Proof. From (2) of the previous section, we have a $G\left(\mathbb{Q}_{p}\right)$-embedding

$$
H_{\xi_{x}, \zeta_{x}} \simeq \widetilde{\mathrm{BS}}\left(\rho_{x}\right) \hookrightarrow \mathcal{L}_{\widehat{\mathcal{H}}\left(K^{p}\right)}\left(M, \mathcal{B}_{x}\right),
$$

where we keep writing $M=\bigotimes_{v \nmid p} \pi_{x, v}^{K_{v}}$. We claim this map is automatically continuous when we equip the $\mathcal{L}$-space with the transformation norm and $H_{\xi_{x}}, \xi_{x}$ with the quotient seminorm induced by $\|\cdot\|_{\xi_{x}, \infty}$. Since $H_{\xi_{x}, \zeta_{x}}$ gets the quotient topology, we just have to check continuity of the inflated map

$$
\mathrm{c}-\operatorname{Ind}_{K}^{G}\left(\xi_{x}\right) \rightarrow H_{\xi_{x}}, \zeta_{x} \hookrightarrow \mathcal{L}_{\widehat{\mathcal{H}}\left(K^{p}\right)}\left(M, \mathcal{B}_{x}\right) .
$$

This is simply Frobenius reciprocity made explicit. In particular, the seminorm on $H_{\xi_{x}}, \zeta_{x}$ is actually a norm (as the kernel of the above map is closed). Therefore, $H_{\xi_{x}, \zeta_{x}}$ is Hausdorff, and $B_{\xi_{x}}, \zeta_{x}$ is its universal completion. That is, there is an isometry with dense image,

$$
H_{\xi_{x}, \zeta_{x}} \hookrightarrow B_{\xi_{x}, \zeta_{x}}
$$

(so $B_{\xi_{x}, \zeta_{x}}$ is nonzero). By continuity of the initial map, it has a unique extension

$$
B_{\xi_{x}, \zeta_{x}} \rightarrow \mathcal{L}_{\widehat{\mathcal{H}}\left(K^{p}\right)}\left(M, \mathcal{B}_{x}\right),
$$

which is continuous (but not necessarily injective) and maps into the completion $\widetilde{\mathrm{BS}}\left(\rho_{x}\right)^{\wedge}$ from Corollary 19, with dense image (but not necessarily onto).

Remark. This fits perfectly with the picture suggested in the papers [Schneider and Teitelbaum 2006; Breuil and Schneider 2007]. If there is a local $p$-adic Langlands correspondence $\rho \mapsto \mathfrak{B}(\rho)$, these references speculate that $B_{\xi, \zeta}$ maps to each $\mathfrak{B}(\rho)$, with dense image, for all crystalline representations $\rho$ of type $(\xi, \zeta)$.

\section{Zariski density of crystalline points}

In general, it is not expected that $\mathcal{B}_{x}^{\mathrm{alg}}$ is dense in $\mathcal{B}_{x}$. In this section, we will adapt (and elaborate on) an argument from Sections 5.3 and 5.4 in [Emerton 2011], which 
builds on ideas of Katz - and which shows the density of crystalline points. This is not needed for this paper, but we include it here for future reference.

7.1. Injectivity of certain modules. We fix a finite extension $L \mid \mathbb{Q}_{p}$, and we will write $\mathcal{O}=\mathcal{O}_{L}$ and $\varpi=\varpi_{L}$, and so on. We will look at locally constant functions $f: Y\left(K^{p}\right) \rightarrow A$ taking values in various finite $\mathcal{O}$-modules $A=\mathcal{O} / \varpi^{s} \mathcal{O}$, where $s$ is a positive integer. These functions form a (discrete) torsion $\mathcal{O}$-module, denoted $H^{0}\left(K^{p}, A\right)$, carrying a natural action of the Hecke algebra $\mathcal{H}_{\mathcal{O}}\left(K^{p}\right)$, and a commuting smooth $G\left(\mathbb{Q}_{p}\right)$-action, which is admissible in the following sense: for every compact open subgroup of $G\left(\mathbb{Q}_{p}\right)$, its invariants form a finite $\mathcal{O}$-module (torsion and finitely generated means finite cardinality, since $A$ is a finite ring).

Lemma 22. Suppose $K^{p}$ is sufficiently small (for example, it suffices that $K_{v}$ has no $p$-torsion for some $v \nmid p)$. Then, for any compact open subgroup $K_{p} \subset G\left(\mathbb{Q}_{p}\right)$, $H^{0}\left(K^{p}, \mathcal{O} / \varpi^{s} \mathcal{O}\right)$ is an injective smooth $\left(\mathcal{O} / \varpi^{s} \mathcal{O}\right)\left[K_{p}\right]$-module for all $s \geq 1$.

Consequently, every direct summand of $H^{0}\left(K^{p}, \mathcal{O} / \varpi^{s} \mathcal{O}\right)$ is an injective ${ }^{4}$ module. Proof. We have to show the exactness of the functor sending a module $M$ to

$$
\operatorname{Hom}_{\mathcal{O}\left[K_{p}\right]}\left(M, H^{0}\left(K^{p}, \mathcal{O} / \varpi^{s} \mathcal{O}\right)\right) .
$$

Here $M$ is an $\mathcal{O}\left[K_{p}\right]$-module with $\varpi^{s} M=0$. Therefore, it has Pontryagin dual

$$
M^{\vee}=\operatorname{Hom}_{\mathcal{O}}(M, L / \mathcal{O})=\operatorname{Hom}_{\mathcal{O}}\left(M, \varpi^{-s} \mathcal{O} / \mathcal{O}\right) \simeq \operatorname{Hom}_{\mathcal{O} / \varpi^{s} \mathcal{O}}\left(M, \mathcal{O} / \varpi^{s} \mathcal{O}\right) .
$$

(Here $M$ is smooth, so we equip it with the discrete topology.) The initial module above can then be identified with that consisting of all functions

$$
f: Y\left(K^{p}\right) \rightarrow M^{\vee}, \quad f(g k)=k^{-1} f(g)
$$

for $k \in K_{p}$. Choosing representatives $g_{i} \in G\left(\mathbb{A}_{f}\right)$ for the finite set $Y\left(K_{p} K^{p}\right)$, and mapping $f$ to the tuple of all $f\left(g_{i}\right)$, then identifies the latter with the direct sum $\bigoplus_{i}\left(M^{\vee}\right)^{\Gamma_{i}}$, where the $\Gamma_{i}$ are certain finite subgroups of $K_{p}$ having prime-to- $p$ order by assumption. This ensures that $(\cdot)^{\Gamma_{i}}$ is exact, by averaging. Also, taking the Pontryagin dual is exact $(L / \mathcal{O}$ is divisible). Finally, as is well known (and easy to check) every summand of an injective module is itself injective.

Examples. Let us first introduce certain finite-type Hecke algebras. For each $K_{p}$, we let $\mathbb{T}\left(K_{p} K^{p}\right)$ denote the image of $\mathfrak{h}^{\circ}=\mathcal{H}_{\mathcal{O}}\left(K^{p}\right)^{\mathrm{sph}}$ in the endomorphism algebra $\operatorname{End}_{\mathcal{O}} H^{0}\left(Y\left(K_{p} K^{p}\right), \mathcal{O}\right)$. Thus $\mathbb{T}\left(K_{p} K^{p}\right)$ is finite free over (the PID) $\mathcal{O}$, and we endow it with the $\varpi$-adic topology. If we have a subgroup $K_{p}^{\prime} \subset K_{p}$, there

\footnotetext{
${ }^{4}$ The injectivity (of modules) addressed here should not be confused with the injectivity of the maps discussed in the remark on p. 209.
} 
is a natural restriction map $\mathbb{T}\left(K_{p}^{\prime} K^{p}\right) \rightarrow \mathbb{T}\left(K_{p} K^{p}\right)$, and we take the limit

$$
\mathbb{T}\left(K^{p}\right):={\overleftarrow{K_{p}}}_{\lim } \mathbb{}\left(K_{p} K^{p}\right) \subset \operatorname{End}_{\mathcal{O}} \tilde{H}^{0}\left(K^{p}\right)^{\circ}
$$

the closure of the image of $\mathcal{H}_{\mathcal{O}}\left(K^{p}\right)^{\text {sph }}$. This defines a reduced, commutative, complete, topological $\mathcal{O}$-algebra. Moreover, $\mathbb{T}\left(K^{p}\right)$ has only finitely many ${ }^{5}$ maximal ideals: they correspond to the maximal ideals of $\mathbb{T}\left(K^{p}\right) \otimes \mathbb{F}$, which is the image of $\mathfrak{h}^{\circ}$ in $\operatorname{End}_{\mathbb{F}} H^{0}\left(K^{p}, \mathbb{F}\right)$. Hence, the maximal ideals are in bijection with the (Galois conjugacy classes of) eigensystems $\mathfrak{h}^{\circ} \rightarrow \mathbb{F}$ which occur in $H^{0}\left(K^{p}, \mathbb{F}\right)$. If $K_{p}$ is any pro- $p$ group, they all must occur in $H^{0}\left(Y\left(K_{p} K^{p}\right), \mathbb{F}\right)$, which is finite-dimensional. Therefore, since $\mathcal{O}$ is complete, we have

$$
\mathbb{T}\left(K^{p}\right) \stackrel{\sim}{\longrightarrow} \prod_{\mathfrak{m}} \mathbb{T}\left(K^{p}\right)_{\mathfrak{m}},
$$

where the product extends over the finitely many maximal ideals $\mathfrak{m} \subset \mathbb{T}\left(K^{p}\right)$, and $\mathbb{T}\left(K^{p}\right)_{\mathfrak{m}}$ denotes the corresponding localization, a complete local $\mathcal{O}$-algebra. (We refer to Chapter 4 of [Darmon et al. 1997] for a discussion of the commutative algebra needed.) We will use this product decomposition as follows: Obviously,

$$
\widetilde{H}^{0}\left(K^{p}\right)^{\circ} / \varpi^{s} \tilde{H}^{0}\left(K^{p}\right)^{\circ} \simeq H^{0}\left(K^{p}, \mathcal{O} / \varpi^{s} \mathcal{O}\right)
$$

carries an action of $\mathbb{T}\left(K^{p}\right)$. This gives rise to a direct sum decomposition,

$$
H^{0}\left(K^{p}, \mathcal{O} / \varpi^{s} \mathcal{O}\right) \stackrel{\sim}{\longrightarrow} \bigoplus_{\mathfrak{m}} H^{0}\left(K^{p}, \mathcal{O} / \varpi^{s} \mathcal{O}\right)_{\mathfrak{m}},
$$

into localized smooth admissible $G\left(\mathbb{Q}_{p}\right)$-submodules over $\mathcal{O} / \varpi^{s} \mathcal{O}$

$$
H^{0}\left(K^{p}, \mathcal{O} / \varpi^{s} \mathcal{O}\right)_{\mathfrak{m}}:=H^{0}\left(K^{p}, \mathcal{O} / \varpi^{s} \mathcal{O}\right) \otimes_{\mathbb{T}\left(K^{p}\right)} \mathbb{T}\left(K^{p}\right)_{\mathfrak{m}},
$$

which are then injective $\left(\mathcal{O} / \varpi^{s} \mathcal{O}\right)\left[K_{p}\right]$-modules for every compact open $K_{p}$.

To connect this to the previous discussion, one could take the maximal ideal $\mathfrak{m}_{x}=\operatorname{ker}\left(\bar{\lambda}_{x}\right)$ for a point $x \in \mathbb{X}(L)$. A priori, this is a maximal ideal in $\mathfrak{h}^{\circ}$, but it is the pull-back of an ideal $\mathfrak{m} \subset \mathbb{T}\left(K^{p}\right)$, since $\bar{\lambda}_{x}$ occurs in tame level $K^{p}$.

7.2. Projective modules over certain Iwasawa algebras. To simplify notation, we write $A=\mathcal{O} / \varpi^{s} \mathcal{O}$ in this section, where $s>0$ is fixed for the moment. We will briefly recall known facts about the Pontryagin duality functor $M \mapsto M^{\vee}$, which sends a discrete $A\left[K_{p}\right]$-module $M$ to the compact

$$
M^{\vee}=\operatorname{Hom}_{\mathcal{O}}(M, L / \mathcal{O}) \simeq \operatorname{Hom}_{A}(M, A) .
$$

${ }^{5}$ The finiteness of the number of cohomological mod $p$ Hecke eigensystems has been proved in much greater generality by Ash and Stevens [1986]. 
If $M$ is smooth, $M=\lim _{\longrightarrow} M^{H}$, with $H$ running over normal open subgroups of $K_{p}$, and therefore its dual $M^{\vee}=\lim _{H}\left(M^{H}\right)^{\vee}$ becomes a module for the Iwasawa algebra

$$
A \llbracket K_{p} \rrbracket:=\lim _{H}^{\lim } A\left[K_{p} / H\right]
$$

Conversely, if $X$ is an $A \llbracket K_{p} \rrbracket$-module, $X / I_{H} X$ becomes a module for $A\left[K_{p} / H\right]$, where $I_{H}$ is the kernel of the natural projection $A \llbracket K_{p} \rrbracket \rightarrow A\left[K_{p} / H\right]$. It follows that $X^{\vee}$ is again a smooth $A\left[K_{p}\right]$-module, since

$$
\left(X^{\vee}\right)^{H} \simeq\left(X / I_{H} X\right)^{\vee} .
$$

Thus, duality sets up a one-to-one correspondence $M \leftrightarrow X$ between smooth discrete $A\left[K_{p}\right]$-modules and compact $A \llbracket K_{p} \rrbracket$-modules which reverses arrows.

Lemma 23. Suppose $M$ is a smooth $A\left[K_{p}\right]$-module, with Pontryagin dual $M^{\vee}$.

(i) $M$ is admissible $\Longleftrightarrow M^{\vee}$ is finitely generated over $A \llbracket K_{p} \rrbracket$.

(ii) $M$ is injective $\Longleftrightarrow M^{\vee}$ is a projective $A \llbracket K_{p} \rrbracket$-module.

Proof. For part (i), if $X$ is finitely generated over $A \llbracket K_{p} \rrbracket$, we deduce that $X / I_{H} X$ is finitely generated over $A\left[K_{p} / H\right]$, which is a ring of finite cardinality. Therefore its dual $M^{H}$ is (physically) finite. For the converse, suppose $M$ is admissible. Then, first of all, $M^{\vee}$ is profinite, so we may apply the "converse" (topological) Nakayama lemma discussed in depth in [Balister and Howson 1997] (specifically, their main theorem in Chapter 3, Section (1), and its corollary): to verify that $M^{\vee}$ is finitely generated over the compact ring $A \llbracket K_{p} \rrbracket$, it suffices to check that $X / I_{H} X$ is finitely generated over $A\left[K_{p} / H\right]$ for some $H$ such that $I_{H}^{n} \rightarrow 0$ as $n \rightarrow \infty$. This limit holds for any pro- $p$-group $H$; see Lemma 3.2 in [Schneider and Teitelbaum 2002], for example. Finiteness of $X / I_{H} X$, or rather its dual $M^{H}$, is admissibility.

For part (ii), use that Pontryagin duality is exact (divisibility of $L / \mathcal{O}$ ). It follows that $\operatorname{Hom}_{A\left[K_{p}\right]}(-, M)$ is exact if and only if $\operatorname{Hom}_{A \llbracket K_{p} \rrbracket}\left(M^{\vee},-\right)$ is exact.

From the last two lemmas, we immediately conclude the following:

Proposition 24. Suppose $K^{p}$ is sufficiently small. Then, for any compact open subgroup $K_{p} \subset G\left(\mathbb{Q}_{p}\right)$, the dual $H^{0}\left(K^{p}, A\right)^{\vee}$ is a projective finitely generated module over $A \llbracket K_{p} \rrbracket$ for all $s \geq 1$. The same is true for any direct summand, such as the localized module $H^{0}\left(K^{p}, A\right)_{\mathfrak{m}}^{\vee}$ for any maximal ideal $\mathfrak{m}$.

For later use, we will record the following fact here. Often, the Iwasawa algebra $A \llbracket K_{p} \rrbracket$ is viewed as a distribution algebra. Indeed, there is a natural pairing with the continuous (that is, locally constant) functions $\mathcal{C}\left(K_{p}, A\right)$.

Lemma 25. $A \llbracket K_{p} \rrbracket \stackrel{\sim}{\longrightarrow} \mathcal{C}\left(K_{p}, A\right)^{\vee}$, as modules over $A \llbracket K_{p} \rrbracket$. 
Proof. For any normal open subgroup $H$, there is a canonical integration pairing,

$$
\mathcal{C}\left(K_{p} / H, A\right) \times A\left[K_{p} / H\right] \rightarrow A, \quad(f, \mu) \mapsto \sum_{k \in K_{p} / H} f(k) \mu(k),
$$

which is nondegenerate, and therefore defines an isomorphism

$$
A\left[K_{p} / H\right] \stackrel{\sim}{\longrightarrow} \mathcal{C}\left(K_{p} / H, A\right)^{\vee}, \quad \mu \mapsto(-, \mu) .
$$

This is easily checked to preserve the $A\left[K_{p} / H\right]$-module structures on both sides. Moreover, as $H$ varies, these isomorphisms are compatible with the transition maps. Passing to the projective limit $\lim _{H}$ gives the lemma.

In other words, $\mathcal{C}\left(K_{p}, A\right) \leftrightarrow A \llbracket K_{p} \rrbracket$ under the correspondence discussed above.

7.3. Local Iwasawa algebras of pro-p-groups. A local ring is a (possibly noncommutative) ring $R$ whose Jacobson radical $J(R)$ is a two-sided maximal ideal $\mathfrak{m}_{R}$. In other words, there is a unique maximal left ideal, and a unique maximal right ideal, and they coincide. Nakayama's lemma even holds for noncommutative local rings, as is easily checked. In particular, a finitely generated projective $R$-module is free, a key fact we will make use of below, by taking $R$ to be the Iwasawa algebra of a pro- $p$-group, which turns out to be local. We first assemble the following well-known facts.

Lemma 26. Let $K_{p}$ be a pro-p-group, and let $A$ be any $p$-ring (that is, its cardinality is a finite power of $p$, such as for $\left.A=\mathcal{O} / \varpi^{s} \mathcal{O}\right)$. Then:

(1) Let $M$ be a left $A \llbracket K_{p} \rrbracket$-module, and $H \subset K_{p}$ an open normal subgroup. Then $M / I_{H} M$ has a nonzero $K_{p}$-invariant element if $M \neq I_{H} M$.

(2) $K_{p}$ acts trivially on any simple left $A \llbracket K_{p} \rrbracket$-module.

(3) $I_{K_{p}} \subset J\left(A \llbracket K_{p} \rrbracket\right)$.

(4) A local $\Longrightarrow A \llbracket K_{p} \rrbracket$ local. (Furthermore, $J\left(A \llbracket K_{p} \rrbracket\right)=\mathfrak{m}_{A}+I_{K_{p}}$.)

(The same is true when left modules are replaced by right modules.)

Proof. This is all standard. We cannot resist briefly outlining the argument. For (1) it is clearly enough to show that a $p$-group $K_{p}$ fixes a nonzero element of any $A\left[K_{p}\right]$-module $M \neq 0$. This is basic group action theory; the fact that $A$ is a $p$-ring allows us to count fixed points modulo $p$. For (2), if $M$ is a simple left $A \llbracket K_{p} \rrbracket$-module, we must have $I_{H} M=M$ or $I_{H} M=0$ for all $H$. There must be some $H$ for which $I_{H} M \neq M$, since $M \neq 0$ is the inverse limit of all quotients $M / I_{H} M$. Now (1) shows that $M^{K_{p}} \neq 0$. By simplicity, $K_{p}$ acts trivially on $M$. For (3) just use that $I_{K_{p}}$ is generated by elements $k-1$ with $k \in K_{p}$. We see from (2) that $I_{K_{p}}$ acts trivially on any simple left $A \llbracket K_{p} \rrbracket$-module, and therefore, by the very definition of the Jacobson radical, we have the inclusion as claimed. Now 
(4) is immediate from (3). Indeed any maximal left ideal of $A \llbracket K_{p} \rrbracket$ must be the pull-back of $\mathfrak{m}_{A}$ under the augmentation map.

Together with Proposition 24, we will apply this to $A \llbracket K_{p} \rrbracket$ with $A=\mathcal{O} / \varpi^{s} \mathcal{O}$.

Proposition 27. Suppose $K^{p}$ is sufficiently small, and let $K_{p} \subset G\left(\mathbb{Q}_{p}\right)$ be an open pro-p-group. Then there exists an integer $r>0$ such that

$$
\tilde{H}^{0}\left(K^{p}\right)^{\circ} \simeq \mathcal{C}\left(K_{p}, \mathcal{O}\right)^{r}
$$

as $\mathcal{O}\left[K_{p}\right]$-modules. Moreover, for any maximal ideal $\mathfrak{m} \subset \mathbb{T}\left(K^{p}\right)$, the localization $\widetilde{H}^{0}\left(K^{p}\right)_{\mathfrak{m}}^{\circ}$ sits as a topologically direct summand.

Proof. Since $A \llbracket K_{p} \rrbracket$ is local, Nakayama's lemma (and Proposition 24) tells us that $H^{0}\left(K^{p}, A\right)^{\vee}$ is a free $A \llbracket K_{p} \rrbracket$-module, of finite rank $r_{s}$, say. Taking the Pontryagin dual then yields an isomorphism of smooth $A\left[K_{p}\right]$-modules

$$
H^{0}\left(K^{p}, \mathcal{O} / \varpi^{s} \mathcal{O}\right) \simeq \mathcal{C}\left(K_{p}, \mathcal{O} / \varpi^{s} \mathcal{O}\right)^{r_{s}} .
$$

Now, we claim that $r_{s}$ is in fact independent of $s>0$ (and we will just write $r$ instead of $r_{s}$ ). To see this, scale both sides of the isomorphism by $\varpi$, compare the corresponding quotients, take $H$-invariants for some $H$, and compare dimensions over $\mathbb{E}$. This shows that $r_{s}=r_{1}$. This allows us to take the inverse limit over $s$ to obtain an isomorphism of modules over $\mathcal{O}\left[K_{p}\right]$

$$
\widetilde{H}^{0}\left(K^{p}\right)^{\circ}={\underset{\lim }{s}}_{H^{0}}\left(K^{p}, \mathcal{O} / \varpi^{s} \mathcal{O}\right) \simeq \mathcal{C}\left(K_{p}, \mathcal{O}\right)^{r},
$$

or, in other words, an isometry $\widetilde{H}^{0}\left(K^{p}\right) \simeq \mathcal{C}\left(K_{p}, L\right)^{r}$ of Banach representations of $K_{p}$. Finally, we may localize at any maximal ideal $\mathfrak{m} \subset \mathbb{T}\left(K^{p}\right)$ and realize $\tilde{H}^{0}\left(K^{p}\right)_{\mathfrak{m}}^{\circ}$ as a (topologically) direct summand of $\mathcal{C}\left(K_{p}, \mathcal{O}\right)^{r}$.

7.4. Mahler expansions and full level at p. Proposition 27 already shows that the algebraic vectors are dense in $\widetilde{H}^{0}\left(K^{p}\right)$ (by employing Mahler expansions, as below). In fact, this is even true for the unit ball $\widetilde{H}^{0}\left(K^{p}\right)^{\circ}$. However, we can be more precise, and prove density of the smaller set of $G\left(\mathbb{Z}_{p}\right)$-locally algebraic vectors, those $f \in \widetilde{H}^{0}\left(K^{p}\right)$ such that $\left\langle G\left(\mathbb{Z}_{p}\right) f\right\rangle$ is an algebraic representation of $G\left(\mathbb{Z}_{p}\right)$ :

Proposition 28. $\tilde{H}^{0}\left(K^{p}\right)^{G\left(\mathbb{Z}_{p}\right) \text {-alg }}$ is dense in $\widetilde{H}^{0}\left(K^{p}\right)\left(\right.$ similarly for $\left.\widetilde{H}^{0}\left(K^{p}\right)_{\mathfrak{m}}\right)$.

Proof. Pick an open, normal pro- $p$-subgroup $K_{p} \subset G\left(\mathbb{Z}_{p}\right)$. From Proposition 27, we have an isometry $\widetilde{H}^{0}\left(K^{p}\right) \simeq \mathcal{C}\left(K_{p}, L\right)^{r}$ of Banach space representations of $K_{p}$. We take the topological dual space $\mathcal{L}(-, L)$ on both sides, and get

$$
\tilde{H}^{0}\left(K^{p}\right)^{\vee} \simeq L \llbracket K_{p} \rrbracket^{r}, \quad L \llbracket K_{p} \rrbracket:=L \otimes_{\mathcal{O}} \mathcal{O} \llbracket K_{p} \rrbracket .
$$


Here $L \llbracket K_{p} \rrbracket$ is identified with the distribution algebra $\mathcal{C}\left(K_{p}, L\right)^{\vee}$ (equipped with the bounded-weak topology) as in [Schneider and Teitelbaum 2002], so $\widetilde{H}^{0}\left(K^{p}\right)^{\vee}$ is a free $L \llbracket K_{p} \rrbracket$-module of rank $r$. It follows that $\widetilde{H}^{0}\left(K^{p}\right)^{\vee}$ is projective over $L \llbracket G\left(\mathbb{Z}_{p}\right) \rrbracket$, as

$$
\left.\operatorname{Hom}_{L \llbracket G\left(\mathbb{Z}_{p}\right) \rrbracket}\left(\tilde{H}^{0}\left(K^{p}\right)^{\vee},-\right)=\operatorname{Hom}_{L \llbracket K_{p} \rrbracket}\left(\tilde{H}^{0}\left(K^{p}\right)^{\vee},-\right)\right)^{G\left(\mathbb{Z}_{p}\right) / K_{p}}
$$

is exact: $\widetilde{H}^{0}\left(K^{p}\right)^{\vee}$ is projective over $L \llbracket K_{p} \rrbracket$, and taking invariants under the finite group $G\left(\mathbb{Z}_{p}\right) / K_{p}$ is exact, by averaging (we are in characteristic zero). Being projective, $\widetilde{H}^{0}\left(K^{p}\right)^{\vee}$ is a direct summand of a free module (of finite rank by finite generation). That is, there is an $s>0$, and a submodule $Z$, such that

$$
\tilde{H}^{0}\left(K^{p}\right)^{\vee} \oplus Z \simeq L \llbracket G\left(\mathbb{Z}_{p}\right) \rrbracket^{s} .
$$

Again, undoing the dual, and invoking Corollary 2.2 and Theorem 3.5 in [Schneider and Teitelbaum 2002],

$$
\widetilde{H}^{0}\left(K^{p}\right) \oplus Z^{\vee} \simeq \mathcal{C}\left(G\left(\mathbb{Z}_{p}\right), L\right)^{s} .
$$

Comparing the $G\left(\mathbb{Z}_{p}\right)$-algebraic vectors on both sides, we see that it suffices to show that they are dense in $\mathcal{C}\left(G\left(\mathbb{Z}_{p}\right), L\right)$. Now, topologically, we identify $G\left(\mathbb{Z}_{p}\right) \simeq \prod_{v \mid p} \mathrm{GL}_{n}\left(\mathcal{O}_{\tilde{v}}\right)$ with a closed-open subset of $\prod_{v \mid p} \mathcal{O}_{\tilde{v}}^{n^{2}} \simeq \mathbb{Z}_{p}^{t}$, where we have introduced $t=[F: \mathbb{Q}] n^{2}$. Any continuous function on $G\left(\mathbb{Z}_{p}\right)$ therefore extends (nonuniquely) to a continuous function on $\mathbb{Z}_{p}^{t}$, which has a (multivariable) Mahler power series expansion [1958], which shows that the polynomials are dense in $\mathcal{C}\left(\mathbb{Z}_{p}^{t}, L\right)$. Finally, observe that polynomials obviously restrict to $G\left(\mathbb{Z}_{p}\right)$-algebraic functions in $\mathcal{C}\left(G\left(\mathbb{Z}_{p}\right), L\right)$. At last, localize at $\mathfrak{m}$.

7.5. Density and locally algebraic vectors. Following [Emerton 2011, Section 5.4], we deduce from the previous proposition that "crystalline points are dense".

Corollary 29. The submodule $\bigoplus_{\lambda \in C} \widetilde{H}^{0}\left(K^{p}\right)^{\mathrm{alg}}[\lambda]$ is dense in $\widetilde{H}^{0}\left(K^{p}\right)$, where $C$ denotes the collection of Hecke eigensystems $\lambda: \mathcal{H}\left(K^{p}\right)^{\mathrm{sph}} \rightarrow \overline{\mathbb{Q}}_{p}$ associated with an automorphic $\pi$, which is unramified at $p$ (and of tame level $K^{p}$ ). Thus, the set of points $\operatorname{ker}(\lambda)$, with $\lambda \in C$, are Zariski dense in $\operatorname{Spec} \mathbb{T}\left(K^{p}\right)[1 / p]$.

Proof. First off, recall from Section 3.2 that we have a decomposition

$$
\tilde{H}^{0}\left(K^{p}\right)^{\mathrm{alg}}=\bigoplus_{W} W \otimes H^{0}\left(K^{p}, \mathcal{V}_{\breve{W}}\right)=\bigoplus_{W} \bigoplus_{\pi: \pi_{\infty} \simeq W} m_{G}(\pi)\left(W \otimes \pi_{f}^{K^{p}}\right) .
$$

In particular,

$$
\tilde{H}^{0}\left(K^{p}\right)^{G\left(\mathbb{Z}_{p}\right)-\text { alg }}=\bigoplus_{W} \bigoplus_{\pi: \pi_{\infty} \simeq W} m_{G}(\pi)\left(W \otimes \pi_{p}^{G\left(\mathbb{Z}_{p}\right)} \otimes\left(\pi_{f}^{p}\right)^{K^{p}}\right),
$$


which is dense in $\widetilde{H}^{0}\left(K^{p}\right)$. A fortiori, so is the $G\left(\mathbb{Q}_{p}\right)$-submodule it generates,

$$
\left\langle\tilde{H}^{0}\left(K^{p}\right)^{G\left(\mathbb{Z}_{p}\right) \text {-alg }}\right\rangle_{G\left(\mathbb{Q}_{p}\right)}=\bigoplus_{W} \bigoplus_{\substack{\pi: \pi_{\infty} \simeq W, \pi_{p}^{G(\mathbb{Z} p)} \neq 0}} m_{G}(\pi)\left(W \otimes \pi_{f}^{K^{p}}\right) .
$$

We decompose the latter into eigenspaces for the action $\mathcal{H}\left(K^{p}\right)^{\mathrm{sph}}$. That is, as

$$
\left\langle\widetilde{H}^{0}\left(K^{p}\right)^{G\left(\mathbb{Z}_{p}\right) \text {-alg }}\right\rangle_{G\left(\mathbb{Q}_{p}\right)}=\bigoplus_{\lambda} \tilde{H}^{0}\left(K^{p}\right)^{\mathrm{alg}}[\lambda]
$$

where $\lambda: \mathcal{H}\left(K^{p}\right)^{\mathrm{sph}} \rightarrow \overline{\mathbb{Q}}_{p}$ runs over all eigensystems of the form $\lambda=\lambda_{\pi}$ for some automorphic $\pi$, of tame level $K^{p}$, which is unramified at $p$ (and of some weight $W)$. Thus, elements of $\bigcap_{\lambda \in C} \operatorname{ker}(\lambda)$ act trivially on $\widetilde{H}^{0}\left(K^{p}\right)$.

\section{Acknowledgements}

I wish to acknowledge the impact of the visions of Breuil and Emerton on this work. I would like to thank both of them for discreetly pointing out some embarrassing misconceptions of mine. Conversations and correspondence with Chojecki, Dospinescu, Helm, Herzig, Newton, Ramakrishnan, and Shin have been a great help and a source of inspiration. I am grateful to Mínguez for clarifying Corollary 4.2 of [Mínguez 2011] to me, and for pointing to the reference [Clozel et al. 2011]. Thanks are due to Caraiani, for making a preliminary version of [Caraiani 2012] available to me before making it public. Finally, I would like to convey my sincere thanks to the referee for a meticulous reading and thoughtful feedback.

\section{References}

[Ash and Stevens 1986] A. Ash and G. Stevens, "Cohomology of arithmetic groups and congruences between systems of Hecke eigenvalues", J. Reine Angew. Math. 365 (1986), 192-220. MR 87i:11069 Zbl 0596.10026

[Balister and Howson 1997] P. N. Balister and S. Howson, "Note on Nakayama's lemma for compact $\Lambda$-modules”, Asian J. Math. 1:2 (1997), 224-229. MR 99f:16047 Zbl 0904.16019

[Barnet-Lamb et al. 2014] T. Barnet-Lamb, T. Gee, D. Geraghty, and R. Taylor, "Local-global compatibility for $l=p$, II", Ann. Sci. École Norm. Sup. (4) 47:1 (2014), 165-179. MR 3205603 Zbl 06324769

[Bellaïche and Chenevier 2009] J. Bellaïche and G. Chenevier, Families of Galois representations and Selmer groups, Astérisque 324, Société Mathématique de France, Paris, 2009. MR 2011m:11105 Zbl 1192.11035

[Berger 2011] L. Berger, "La correspondance de Langlands locale $p$-adique pour $\mathrm{GL}_{2}\left(\mathbb{Q}_{p}\right)$ )", pp. 157-180 in Séminaire Bourbaki: Vol. 2009/2010, Exposés 1012-1026, Astérisque 339, Société Mathématique de France, Paris, 2011. MR 2012k:11186

[Bergeron and Clozel 2005] N. Bergeron and L. Clozel, Spectre automorphe des variétés hyperboliques et applications topologiques, Astérisque 303, Société Mathématique de France, Paris, 2005. MR 2007j:22031 Zbl 1098.11035 
[Breuil 2010] C. Breuil, "The emerging $p$-adic Langlands programme", pp. 203-230 in Proceedings of the International Congress of Mathematicians (Hyderabad, 2010), vol. 2, edited by R. Bhatia et al., Hindustan Book Agency, New Delhi, 2010. MR 2012k:22024 Zbl 05971096

[Breuil and Schneider 2007] C. Breuil and P. Schneider, "First steps towards $p$-adic Langlands functoriality", J. Reine Angew. Math. 610 (2007), 149-180. MR 2009f:11147 Zbl 1180.11036

[Caraiani 2012] A. Caraiani, "Local-global compatibility and the action of monodromy on nearby cycles”, Duke Math. J. 161:12 (2012), 2311-2413. MR 2972460 Zbl 06095601

[Caraiani et al. 2014] A. Caraiani, M. Emerton, T. Gee, D. Geraghty, V. Paskunas, and S. W. Shin, "Patching and the $p$-adic local Langlands correspondence", preprint, 2014. arXiv 1310.0831

[Chenevier 2004] G. Chenevier, "Familles $p$-adiques de formes automorphes pour GL ${ }_{n}$ ", J. Reine Angew. Math. 570 (2004), 143-217. MR 2006b:11046 Zbl 1093.11036

[Chenevier 2009] G. Chenevier, "Une application des variétés de Hecke des groupes unitaires”, preprint, 2009, http://gaetan.chenevier.perso.math.cnrs.fr/articles/famgal.pdf.

[Clozel et al. 2011] L. Clozel, M. Harris, and J.-P. Labesse, "Endoscopic transfer", pp. 475-496 in On the stabilization of the trace formula, edited by L. Clozel et al., Stabilization of the Trace Formula, Shimura Varieties, and Arithmetic Applications 1, International Press, Somerville, MA, 2011. MR 2856382

[Colmez and Fontaine 2000] P. Colmez and J.-M. Fontaine, "Construction des représentations padiques semi-stables”, Invent. Math. 140:1 (2000), 1-43. MR 2001g:11184 Zbl 1010.14004

[Darmon et al. 1997] H. Darmon, F. Diamond, and R. Taylor, "Fermat's last theorem", pp. 2-140 in Elliptic curves, modular forms \& Fermat's last theorem, 2nd ed. (Hong Kong, 1993), edited by J. H. Coates and S.-T. Yau, International Press, Cambridge, MA, 1997. MR 99d:11067b Zbl 0877.11035

[Emerton 2006a] M. Emerton, "Jacquet modules of locally analytic representations of $p$-adic reductive groups, I: Construction and first properties", Ann. Sci. École Norm. Sup. (4) 39:5 (2006), 775-839. MR 2008c:22013 Zbl 1117.22008

[Emerton 2006b] M. Emerton, "On the interpolation of systems of eigenvalues attached to automorphic Hecke eigenforms”, Invent. Math. 164:1 (2006), 1-84. MR 2007k:22018 Zbl 1090.22008

[Emerton 2007] M. Emerton, "Jacquet modules of locally analytic representations of $p$-adic reductive groups, II: The relation to parabolic induction", preprint, 2007, http://www.math.uchicago.edu/ emerton/pdffiles/jacquet-two.pdf. To appear in J. Inst. Math. Jussieu.

[Emerton 2011] M. Emerton, "Local-global compatibility in the $p$-adic Langlands programme for $\mathrm{GL}_{2 / \mathbb{Q}}$ ”, preprint, 2011, http://www.math.uchicago.edu/ emerton/pdffiles/lg.pdf.

[Emerton and Gee 2013] M. Emerton and T. Gee, " $p$-adic Hodge-theoretic properties of étale cohomology with mod $p$ coefficients, and the cohomology of Shimura varieties", preprint, 2013. arXiv 1203.4963

[Fontaine and Mazur 1995] J.-M. Fontaine and B. Mazur, "Geometric Galois representations", pp. 41-78 in Elliptic curves, modular forms, \& Fermat's last theorem (Hong Kong, 1993), edited by J. H. Coates and S.-T. Yau, Series in Number Theory 1, International Press, Cambridge, MA, 1995. MR 96h:11049 Zbl 0839.14011

[Fontaine and Rapoport 2005] J.-M. Fontaine and M. Rapoport, "Existence de filtrations admissibles sur des isocristaux”, Bull. Soc. Math. France 133:1 (2005), 73-86. MR 2005m:14032 Zbl 1073.14025

[Harris and Taylor 2001] M. Harris and R. Taylor, The geometry and cohomology of some simple Shimura varieties, Annals of Mathematics Studies 151, Princeton University Press, 2001. MR 2002m:11050 Zbl 1036.11027

[Hu 2009] Y. Hu, "Normes invariantes et existence de filtrations admissibles", J. Reine Angew. Math. 634 (2009), 107-141. MR 2011c:11177 Zbl 1214.11132 
[Kaletha et al. 2014] T. Kaletha, A. Mínguez, S. W. Shin, and P.-J. White, "Endoscopic classification of representations: inner forms of unitary groups", preprint, 2014. arXiv 1409.3731

[Katz 1978] N. M. Katz, “ $p$-adic L-functions for CM fields”, Invent. Math. 49:3 (1978), 199-297. MR 80h:10039 Zbl 0417.12003

[Lazarus 2000] X. Lazarus, Module universel non ramifié pour un groupe réductif p-adique, $\mathrm{Ph} . \mathrm{D}$. thesis, Université Paris-Sud, 2000.

[Loeffler 2011] D. Loeffler, "Overconvergent algebraic automorphic forms", Proc. Lond. Math. Soc. (3) 102:2 (2011), 193-228. MR 2012f:11098 Zbl 1232.11056

[Mahler 1958] K. Mahler, "An interpolation series for continuous functions of a $p$-adic variable", $J$. Reine Angew. Math. 199 (1958), 23-34. MR 20 \#2321 Zbl 0080.03504

[Mínguez 2011] A. Mínguez, "Unramified representations of unitary groups", pp. 389-410 in On the stabilization of the trace formula, edited by L. Clozel et al., Stabilization of the Trace Formula, Shimura Varieties, and Arithmetic Applications 1, International Press, Somerville, MA, 2011. MR 2856377

[Mok 2014] C. P. Mok, Endoscopic classification of representations of quasi-split unitary groups, Memoirs of the American Mathematical Society 235:1108, American Mathematical Society, Providence, RI, online publication October 2014. In press.

[Schneider 2002] P. Schneider, Nonarchimedean functional analysis, Springer, Berlin, 2002. MR 2003a:46106 Zbl 0998.46044

[Schneider and Teitelbaum 2001] P. Schneider and J. Teitelbaum, " $U(\mathfrak{g})$-finite locally analytic representations", Represent. Theory 5 (2001), 111-128. MR 2002e:22023 Zbl 1028.17007

[Schneider and Teitelbaum 2002] P. Schneider and J. Teitelbaum, "Banach space representations and Iwasawa theory", Israel J. Math. 127 (2002), 359-380. MR 2003c:22026 Zbl 1006.46053

[Schneider and Teitelbaum 2006] P. Schneider and J. Teitelbaum, "Banach-Hecke algebras and p-adic Galois representations", Doc. Math. Extra Volume (2006), 631-684. MR 2008b:11126 Zbl 1140.11026

[Sorensen 2013] C. M. Sorensen, "A proof of the Breuil-Schneider conjecture in the indecomposable case”, Ann. Math. (2) 177:1 (2013), 367-382. MR 2999043 Zbl 06146422

[Taylor 1991] R. Taylor, "Galois representations associated to Siegel modular forms of low weight", Duke Math. J. 63:2 (1991), 281-332. MR 92j:11044 Zbl 0810.11033

[Vignéras 2004] M.-F. Vignéras, “On highest Whittaker models and integral structures”, pp. 773-801 in Contributions to automorphic forms, geometry, and number theory, edited by H. Hida et al., Johns Hopkins University Press, Baltimore, MD, 2004. MR 2006b:11048 Zbl 1084.11023

[Vignéras 2010] M.-F. Vignéras, "Banach $l$-adic representations of $p$-adic groups", pp. 1-11 in Représentations p-adiques de groupes p-adiques, II: Représentations de $\mathrm{GL}_{2}\left(\mathbb{Q}_{p}\right)$ et $(\varphi, \Gamma)$ modules, edited by L. Berger et al., Astérisque 330, Société Mathématique de France, Paris, 2010. MR 2011g:11102 Zbl 1232.11062

[White 2012] P.-J. White, “Tempered automorphic representations of the unitary group", preprint, 2012. arXiv 1106.1127

Received May 7, 2014.

Claus M. Sorensen

DEPARTMENT OF MATHEMATICS

UCSD

9500 GILMAN DR.

LA JOLLA, CA 92093

UNITED STATES

csorensen@ucsd.edu 


\title{
PACIFIC JOURNAL OF MATHEMATICS
}

\author{
msp.org/pjm
}

Founded in 1951 by E. F. Beckenbach (1906-1982) and F. Wolf (1904-1989)

\section{EDITORS}

Don Blasius (Managing Editor)

Department of Mathematics

University of California

Los Angeles, CA 90095-1555

blasius@math.ucla.edu

\author{
Paul Balmer \\ Department of Mathematics \\ University of California \\ Los Angeles, CA 90095-1555 \\ balmer@math.ucla.edu \\ Robert Finn \\ Department of Mathematics \\ Stanford University \\ Stanford, CA 94305-2125 \\ finn@math.stanford.edu \\ Sorin Popa \\ Department of Mathematics \\ University of California \\ Los Angeles, CA 90095-1555 \\ popa@math.ucla.edu
}

\author{
Vyjayanthi Chari \\ Department of Mathematics \\ University of California \\ Riverside, CA 92521-0135 \\ chari@math.ucr.edu \\ Kefeng Liu \\ Department of Mathematics \\ University of California \\ Los Angeles, CA 90095-1555 \\ liu@math.ucla.edu \\ Jie Qing \\ Department of Mathematics \\ University of California \\ Santa Cruz, CA 95064 \\ qing@ cats.ucsc.edu
}

\section{PRODUCTION}

Silvio Levy, Scientific Editor, production@msp.org

\section{SUPPORTING INSTITUTIONS}

ACADEMIA SINICA, TAIPEI

CALIFORNIA INST. OF TECHNOLOGY

INST. DE MATEMÁTICA PURA E APLICADA

KEIO UNIVERSITY

MATH. SCIENCES RESEARCH INSTITUTE

NEW MEXICO STATE UNIV.

OREGON STATE UNIV.

\author{
STANFORD UNIVERSITY \\ UNIV. OF BRITISH COLUMBIA \\ UNIV. OF CALIFORNIA, BERKELEY \\ UNIV. OF CALIFORNIA, DAVIS \\ UNIV. OF CALIFORNIA, LOS ANGELES \\ UNIV. OF CALIFORNIA, RIVERSIDE \\ UNIV. OF CALIFORNIA, SAN DIEGO \\ UNIV. OF CALIF., SANTA BARBARA
}

\author{
Daryl Cooper \\ Department of Mathematics \\ University of California \\ Santa Barbara, CA 93106-3080 \\ cooper@math.ucsb.edu \\ Jiang-Hua Lu \\ Department of Mathematics \\ The University of Hong Kong \\ Pokfulam Rd., Hong Kong \\ jhlu@maths.hku.hk \\ Paul Yang \\ Department of Mathematics \\ Princeton University \\ Princeton NJ 08544-1000 \\ yang@math.princeton.edu
}

These supporting institutions contribute to the cost of publication of this Journal, but they are not owners or publishers and have no responsibility for its contents or policies.

See inside back cover or msp.org/pjm for submission instructions.

The subscription price for 2015 is US \$420/year for the electronic version, and \$570/year for print and electronic.

Subscriptions, requests for back issues and changes of subscribers address should be sent to Pacific Journal of Mathematics, P.O. Box 4163, Berkeley, CA 94704-0163, U.S.A. The Pacific Journal of Mathematics is indexed by Mathematical Reviews, Zentralblatt MATH, PASCAL CNRS Index, Referativnyi Zhurnal, Current Mathematical Publications and Web of Knowledge (Science Citation Index).

The Pacific Journal of Mathematics (ISSN 0030-8730) at the University of California, c/o Department of Mathematics, 798 Evans Hall \#3840, Berkeley, CA 94720-3840, is published twelve times a year. Periodical rate postage paid at Berkeley, CA 94704, and additional mailing offices. POSTMASTER: send address changes to Pacific Journal of Mathematics, P.O. Box 4163, Berkeley, CA 94704-0163.

PJM peer review and production are managed by EditFLOW ${ }^{\circledR}$ from Mathematical Sciences Publishers.

\section{PUBLISHED BY}

\section{mathematical sciences publishers \\ nonprofit scientific publishing}

http://msp.org/

(C) 2015 Mathematical Sciences Publishers 


\section{PACIFIC JOURNAL OF MATHEMATICS}

Volume $275 \quad$ No. $1 \quad$ May 2015

Constant-speed ramps

OSCAR M. PERDOMO

Surfaces in $\mathbb{R}_{+}^{3}$ with the same Gaussian curvature induced by the

Euclidean and hyperbolic metrics

Nilton BARRoso and PEDRO RoITMAN

Cohomology of local systems on the moduli of principally polarized abelian surfaces

DAN PETERSEN

On certain dual $q$-integral equations

Ola A. Ashour, Mourad E. H. Ismail and Zeinab S.

MANSOUR

On a conjecture of Erdôs and certain Dirichlet series

TAPAS CHATTERJEE and M. RAM MURTY

Normal forms for CR singular codimension-two Levi-flat submanifolds

XiAnGHONG GONG and JiŘí LEBL

Measurements of Riemannian two-disks and two-spheres

FLORENT BALACHEFF

Harmonic maps from $\mathbb{C}^{n}$ to Kähler manifolds

JIANMING WAN

Eigenvarieties and invariant norms

Claus M. Sorensen

The Heegaard distances cover all nonnegative integers 